\title{
Intrinsic Structure and Dynamics of the
}

\section{Water/Nitrobenzene Interface}

\author{
Miguel Jorge*, M. Natália D. S. Cordeiro* \\ REQUIMTE, Faculdade de Ciências, Universidade do Porto, Rua do Campo Alegre, 687, 4169- \\ 007 Porto, Portugal
}

Email addresses: miguel.jorge@fc.up.pt; ncordeir@fc.up.pt

Title Running Head: Intrinsic properties of the water/NB interface

\begin{abstract}
In this paper we present results of a detailed and systematic molecular dynamics study of the water/nitrobenzene interface. Using a simple procedure to eliminate fluctuations of the interface position, we are able to obtain true intrinsic profiles for several properties (density, hydrogen bonds, molecular orientation, etc.) in the direction perpendicular to the interfacial plane. Our results show that both water and organic interfacial molecules form a tightly packed layer oriented parallel to the interface, with reduced mobility in the perpendicular direction. Beyond this layer, water quickly restores its bulk structure, while nitrobenzene exhibits structural anisotropies that extend further into the bulk region. Water molecules that protrude farthest into the organic phase point one hydrogen atom in the direction perpendicular to the interface, forming a hydrogen bond with a nitrobenzene oxygen. By fitting both the global and intrinsic
\end{abstract}


density profiles, we obtain estimates for the total and intrinsic interface widths, respectively. These are combined with capillary wave theory to produce a self-consistent method for the calculation of the interfacial tension. Values calculated using this method are in very good agreement with direct calculations from the components of the pressure tensor.

Key words: liquid/liquid interface; surface tension; density profiles; molecular orientation; diffusion coefficient; ion transfer.

\section{Introduction}

Interfaces between water and immiscible organic liquids are ubiquitous in nature, and are important in a wide variety of chemical, physical and biological processes, such as phase transfer catalysis, liquid-liquid extraction and drug delivery ${ }^{1}$. Understanding of these processes relies on fundamental knowledge at the molecular level of the structural and dynamic characteristics of the interface itself. Nitrobenzene was chosen as the organic liquid because it is widely employed in electrochemical studies at interfaces ${ }^{2-5}$, but has received relatively little attention from the theoretical point of view. In 1998, Michael and Benjamin ${ }^{6}$ have presented an MD study of the water/nitrobenzene interface, where they analyzed the structure of the interface and the effect of molecular polarizability on interfacial properties. In a recent letter ${ }^{7}$, these simulations have been extended to help explain X-ray reflectivity measurements. Since the original paper by Michael and Benjamin ${ }^{6}$, our theoretical understanding of interfacial systems has evolved and new methodologies have appeared ${ }^{8-14}$ that are able to provide a more detailed picture of this interface. In this paper, we make use of such recent developments to present a detailed and systematic characterization of the local structure and dynamics of the water/nitrobenzene interface using molecular dynamics (MD) simulations. In the future, we intend to study the transfer of biologically important molecules (such as drugs and aminoacids) across this liquid/liquid interface. 
Despite recent advances ${ }^{15}$ experimental techniques are still limited when it comes to providing a detailed description of a liquid/liquid interface. This is mainly due to the fluidity of the interface and to its buried nature, which precludes local experimental probing. Molecular simulation techniques, on the other hand, are particularly suited for shedding light on atomiclevel phenomena, and have been widely applied to liquid/liquid interfaces (see review by Benjamin $^{1}$ and references therein). Despite the large number of papers published on this topic since Linse's pioneering work on the water/benzene interface ${ }^{16}$, progress in our fundamental understanding of interfacial properties has been relatively slow, perhaps due to the difficulty in defining the interface itself. This was already recognized by Linse ${ }^{16}$, who employed a method based on dividing the plane parallel to the interface in square meshes of variable degrees of refinement (determined by parameter $N$, the number of squares in each direction). In each section, he determined the limits of each phase and calculated a value for the interfacial thickness. The average thickness was seen to decrease with mesh refinement, suggesting that the interface was molecularly sharp and broadened by thermal fluctuations. Benjamin ${ }^{17}$ later extended this method to measure the average and fluctuations in both interface width and position. In his detailed study of the water/1,2-dichloroethane system, he reached the same conclusions as Linse regarding the structure of this interface. Indeed, a picture of an interface that is relatively sharp on a molecular level, but exhibits corrugations caused by thermal fluctuations (or capillary waves) has emerged from every simulation study of liquid/liquid interfaces using realistic potential models. To our knowledge, the only exception has been a study by Carpenter and Hehre ${ }^{18}$ of the water/hexane interface, but this was later attributed to an incorrect choice of alkane potential parameters ${ }^{19}$.

The nature of the liquid/liquid interface described above has led to efforts aiming to describe it using capillary wave theory $(\mathrm{CWT})^{20,21}$. Most of these efforts rely on a relationship established by CWT between the width of the interface due to capillary wave fluctuations $\left(w_{\mathrm{cw}}\right)$ and the macroscopic interfacial tension $(\gamma)^{21}$ : 


$$
w_{\mathrm{cw}}^{2}=\frac{k_{\mathrm{B}} T}{2 \pi \gamma_{\mathrm{cw}}} \ln \left(\frac{L}{\xi}\right)
$$

where $k_{\mathrm{B}}$ is the Boltzmann constant, $T$ is the temperature, $L$ is the length of the simulation box in the directions parallel to the interfacial plane and $\xi$ is the bulk correlation length. The latter parameter is commonly defined, somewhat ambiguously, as being of the order of the molecular diameter. Equation (1) is obtained by neglecting the effects of gravity, which is a reasonable assumption at the small length scales employed in molecular simulations ${ }^{1}$. This equation was used by several authors ${ }^{6,17,22-26}$ to calculate the interfacial tension, using different methods for estimating $w_{\mathrm{cw}}$ and assuming values for $\xi$ that ranged between 0.4 and $0.9 \mathrm{~nm}$. Reasonable agreement with experiment was sometimes found, but in most cases where the interfacial tension of the simulated system $\left(\gamma_{N}\right)$, calculated by the virial route, was also reported, $\gamma_{\mathrm{cw}}$ was significantly below those values $^{6,17}$. Thus, even though CWT in its original form can qualitatively describe the nature of the interface, it is not always successful at predicting quantitative values of the interfacial tension.

Another important property of interfacial systems that has been widely debated is the density profile. Early simulations of liquid/liquid interfaces produced density profiles, calculated by dividing the system in thin slices parallel to the interface, that exhibited large oscillations extending into the bulk regions $s^{6,16-19,22-24,27,28}$. Such oscillations were tentatively attributed to sampling insufficiencies. Indeed, increasing the system size and the sampling time led to a smoothing of the fluctuations in the bulk regions, but oscillations near the interface remained ${ }^{29}$, which suggests that they are intrinsic to the system. In an attempt to clarify this, Fernandes et al. ${ }^{8}$ calculated density profiles of the water/2-heptanone system relative to a local definition of the interface. This was achieved by applying the method used by Linse $^{16}$ and Benjamin ${ }^{17}$ to determine the limits of each phase, and then calculating the density profile relative to these limits. Using this method, one is able to decouple fluctuations occurring in the interfacial plane from 
those perpendicular to it. The resulting local density profiles showed relatively smooth bulk regions and pronounced oscillations near the interface, more pronounced on the organic side ${ }^{8}$. This method was later applied to other interfaces ${ }^{25,26}$, and oscillatory density profiles were also obtained. Although these results point unequivocally to the existence of an intrinsic density profile that is broadened by thermal fluctuations, it has not yet been shown that the method as it stands does indeed yield this intrinsic profile. A recent paper by Chowdhary and Ladanyi ${ }^{30}$ sheds $^{3}$ further light on this issue. They have adapted a procedure developed by Tarazona and coworkers $^{9-11}$ and calculated the true intrinsic profile for several water/hydrocarbon interfaces. The resulting profiles are qualitatively similar to those of Fernandes et al. ${ }^{8}$, but a critical comparison of both methods was not attempted.

The presence of an interface strongly affects the molecular organization of both phases, which becomes evident when one computes different properties as a function of the distance to the interface. In previous molecular simulation studies of liquid/liquid interfaces, this effect has been observed, for example, in density profiles (as discussed above), molecular packing and orientation, hydrogen-bond formation, and so on. The effect of the interface on the hydrogenbonding structure of water is more or less consensual. In his early study, Linse ${ }^{16}$ observed a decrease in the number of $\mathrm{H}$-bonds per molecule from the bulk region to the interface. However, the total number of nearest neighbors in the first water coordination shell also decreased. The combination of these two effects results in an increase of the percentage of hydrogen-bonded water molecules near the interface. This suggests that interfacial water molecules arrange themselves so as to maximize the possibility of forming hydrogen bonds. This conclusion was corroborated in nearly all subsequent simulation studies ${ }^{6,17,18,22-27,31}$, and supported by observations that radial distribution functions (RDFs) of interfacial water exhibited a similar

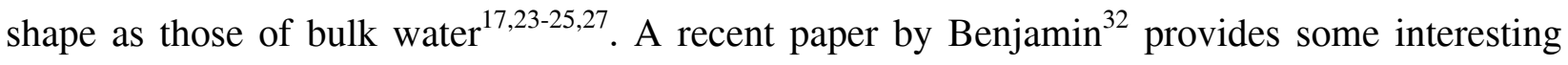
new insights on the dynamics of these hydrogen bonds. 
Studies of the orientation of interfacial water molecules, however, have not been so consensual. Indeed, as discussed by Jedlovszky et al. ${ }^{12}$, some studies led to conclusions that are seemingly inconsistent with each other. Linse ${ }^{16}$ studied the average orientation of the water dipole vector relative to the interface with benzene and concluded that water molecules prefer to align with their dipoles parallel to the interfacial plane. However, van Buuren et al. ${ }^{19}$ concluded that the water dipole vectors are oriented perpendicularly to the interface, in two alternating layers, while Carpenter and Hehre ${ }^{18}$ found no preferential orientation. These discrepancies were resolved when Benjamin ${ }^{17}$ calculated the distributions of the angle between the dipole vector and the interface normal for the water/1,2-dichloroethane system. He observed that in a relatively wide layer adjacent to the interface, the distribution peaked around $\theta=90^{\circ}$, suggesting orientation parallel to the interfacial plane. Similar conclusions were later drawn for other liquid/liquid interfaces ${ }^{6,22-28,33}$. Benjamin ${ }^{17}$ also analyzed the angle between the $\mathrm{H}-\mathrm{H}$ vector of water and the interface normal, concluding that in a thin layer in contact with the organic phase, water molecules had one hydrogen atom pointing toward the organic. The same conclusion was reached by Chang and $\mathrm{Dang}^{27}$ for the water/CCl 4 interface and by Michael and Benjamin ${ }^{6}$ for the water/nitrobenzene interface. However, Jedlovszky et al. ${ }^{12}$ demonstrated that these analyses based on monovariate distributions of angles are clearly insufficient for a full description of the orientation of water molecules. To some extent, the same can be said of studies where this approach was applied to the orientation of organic molecules ${ }^{17,22-27,31}$. Thus, Jedlovszky et al. ${ }^{12}$ proposed a method based on the determination of a bivariate orientation distribution of water angles, thus uniquely defining the orientation of each water molecule. They found two preferential orientations: one is a parallel alignment of the molecular plane with the interfacial plane and the other is characterized by a perpendicular alignment of the molecular plane relative to the interface, with a hydrogen atom pointing directly to the organic phase. The first orientation was prevalent throughout most of the interfacial region, while the second occurred only on the 
organic-rich side of the interface ${ }^{12}$. The same authors then extended their analysis to several water/apolar interfaces (including water vapor and several organic liquids) and showed that these orientational preferences were independent of the nature of the apolar phase ${ }^{34}$.

Finally, we mention another property that has originated some controversy over the years - the interfacial diffusion coefficient. Benjamin ${ }^{17}$ calculated the average water and 1,2dichloroethane diffusion coefficients $(D)$ in slices parallel to the interfacial plane and showed that the diffusion coefficients in the direction parallel to the interface $\left(D_{\mathrm{xy}}\right)$ remains practically constant (with a modest increase in the case of the organic), while that in the direction perpendicular to the interface $\left(D_{\mathrm{z}}\right)$ decreases significantly. $D_{\mathrm{z}}$ was persistently lower than $D_{\mathrm{xy}}$ for both liquids throughout the whole system. However, his results were averaged over rather thick slices but still showed considerable scatter, even in the bulk region. In a later study, Michael and Benjamin $^{6}$ reached similar conclusions for the water/nitrobenzene system. They also observed a small tendency for $D_{\mathrm{xy}}$ to increase, but were unable to resolve it within statistical error. These conclusions are in contrast with a study of an interface between two immiscible Lennard-Jones fluids, where a constant $D_{\mathrm{z}}$ and an increasing $D_{\mathrm{xy}}$ were observed ${ }^{35}$. The discrepancies were attributed by Benjamin ${ }^{17}$ to the higher level of water structuring near the interface. Michael and Benjamin $^{22}$ performed a similar analysis of the water/nonane interface and observed an increase in $D_{\text {xy }}$ and decrease in $D_{\mathrm{z}}$ for both liquids as the interface is approached. Once more, $D_{\mathrm{z}}$ was persistently lower than $D_{\text {xy }}$ throughout the simulation box. Schweighofer and Benjamin ${ }^{23}$, on the contrary, reported a decrease in both $D_{\mathrm{xy}}$ and $D_{\mathrm{z}}$ for interfacial water relative to the bulk. The same conclusion was drawn by Fernandes et al. $^{24}$ in their study of the water/2-heptanone interface, and by Wang et al. ${ }^{33}$ for the water/hexane system. In both cases, $D_{\mathrm{z}}$ was again observed to be lower than $D_{\mathrm{xy}}$ in the entire system. However, Fernandes et al. ${ }^{24}$ reported a small increase in both components of the 2-heptanone diffusion coefficient as the water phase was approached, while Wang et al. ${ }^{33}$ observed a decrease in the hexane diffusion coefficient. One conclusion is 
clear from all these studies - the interface strongly affects the diffusion coefficient. Indeed, all simulations show a strong anisotropy and it was suggested that isotropic diffusion coefficients could only be reached with much larger simulation boxes ${ }^{24}$. The diffusion coefficient is notoriously difficult to calculate with enough accuracy, and it is possible that most of the previous results (particularly the earlier ones) suffer from large statistical errors. Nevertheless, it is quite likely that the direction of change of the two components of $D$ is strongly dependent on the nature of the organic liquid.

This quick review of simulated liquid-liquid interfaces illustrates the insight that has been obtained by applying molecular simulation methods to this problem, but also shows how far we still have to go to reach a detailed understanding of the structure and properties of interfacial systems. In this paper, we make use of some recent advances in the theoretical description of interfaces $^{8-14}$ and perform a detailed and systematic MD study of the water/nitrobenzene interface. We calculate several properties (including most of those discussed above) as a function of distance to the interface, using both a global and a local definition of the interfacial plane. In the next section, we provide some computational details about our simulations. The results section is divided in five parts. Section 3.1 presents our results for the structure of the interface itself and for the density profiles (global and intrinsic). In section 3.2 we attempt to describe the interface using capillary wave theory, and present a self-consistent method for calculating correct values of the interfacial tension. Section 3.3 discusses the influence of the interface on the structure of the fluids and on the hydrogen bond formation, while in section 3.4 we present results for the molecular orientation of both water and organic using a bivariate angle distribution. In section 3.5, we show the variation of the diffusion coefficient of water and nitrobenzene as a function of distance to the interface. Finally, we present our conclusions in section 4. 


\section{Potentials and Methods}

Simulations were performed using the GROMACS MD software package, version $3.3^{36,37}$. The Verlet leapfrog algorithm ${ }^{38}$ was used to integrate the equations of motion, with a timestep of $2 \mathrm{fs}$, and constraints were enforced on all bond lengths using the LINCS algorithm ${ }^{39}$. The simulation box was a cuboid, elongated in the direction perpendicular to the interface $\left(L_{\mathrm{z}}>\right.$ $\left.L_{\mathrm{x}}=L_{\mathrm{y}}=L\right)$. Periodic boundary conditions were used in all three Cartesian directions, giving rise to two distinct water/organic interfaces. All runs were carried out in the $N P_{z} A T$ ensemble ${ }^{28}$. The temperature was kept constant at $298 \mathrm{~K}$ by applying the Nosé-Hoover thermostat ${ }^{40,41}$, with a coupling constant of $1.0 \mathrm{ps}$. The pressure normal to the interfacial plane was kept fixed at 1 bar using the semi-isotropic form of the Parrinello-Rahman barostat ${ }^{42}$, with a coupling constant of 1.0 ps. In practice, $L_{\mathrm{x}}$ and $L_{\mathrm{y}}$ were kept fixed (constant cross-sectional area) while $L_{\mathrm{z}}$ was allowed to fluctuate during the simulation. We have performed simulations for two values of $L$ (2.5 and 3.5 $\mathrm{nm})$, in both cases with a value of $L_{\mathrm{z}}$ around $8.5 \mathrm{~nm}$.

Water was modeled by the SPC/E potential ${ }^{43}$, with rigid bond lengths and angle. For nitrobenzene, we have compared two different potential models, the OPLS all-atom transferable force field ${ }^{44,45}$ and the model of Michael and Benjamin $(M B)^{6}$, specially designed for the nitrobenzene molecule. A previous study has shown that the latter yields slightly worse agreement with experiments than OPLS for most calculated bulk properties, with the exception of the molecular dipole moment ${ }^{46}$. Since this property may have a strong effect on the interface between nitrobenzene and water (a very polar molecule), we have chosen to compare the performance of both models in this study. The total interaction potential between two molecules was thus calculated as the sum of all bonded (angle bending, proper and improper torsions) and non-bonded (Lennard-Jones and Coulomb) terms. Parameters for these models have been presented previously ${ }^{6,46}$. The dispersion interactions were calculated using a twin-range cutoff scheme, with cutoff radii of 1.1 and $1.5 \mathrm{~nm}$, plus a long-range correction term added to both 
energy and pressure. The particle-mesh Ewald method ${ }^{47}$, with a real-space cutoff of $1.1 \mathrm{~nm}$, was applied to handle the electrostatic interactions.

The procedure to construct the initial two-phase configuration was as follows. First, two individual boxes with the same $x$ and $y$ dimensions, containing each of the pure liquids, were constructed and equilibrated. These boxes were then fitted together in the $z$ direction, leaving a small gap between both phases so as to avoid atom overlap (this gap was approxiately $0.5 \mathrm{~nm}$ wide). Next, the whole system was translated in the $z$ direction, using periodic boundary conditions, so that the center of mass of the organic phase corresponded to the geometric center of the simulation box. Finally, the box was equilibrated in the $N P_{\mathrm{z}} A T$ ensemble, until $L_{\mathrm{z}}$ fluctuated about an equilibrium value. Figure 1 shows the result of this procedure - a simulation box containing the organic phase in the center, separated by interfaces from the water phase at its left and right. After the two-phase system was equilibrated, properties were sampled during 5 ns, divided in blocks of 200 ps for averaging purposes.

\section{Figure 1}

\section{Results and Discussion}

\subsection{Interfacial Structure and Density Profiles}

We begin our analysis of the water/nitrobenzene interface by calculating the density profiles normal to the interfacial plane (i.e., in the $z$ direction of our coordinate system - see Figure 1). For each configuration in the sampling stage, we rescaled the $z$ coordinates of each atom by a fixed amount so that the center of mass of the organic phase is located at the origin. This eliminates any possible artifacts due to the translational drift of the interface ${ }^{17}$. Subsequently, we divided the simulation box in slabs of width $0.04 \mathrm{~nm}$ relative to a fixed coordinate frame. In each slab, we calculated the instantaneous density of each species and averaged over all configurations. We have tested different slab widths and concluded that the 
value of $0.04 \mathrm{~nm}$ provides an adequate balance between accuracy of the resulting profiles and low statistical noise. The resulting "global" density profiles are shown in Figure 2 for both nitrobenzene models with a box size of $L=3.5 \mathrm{~nm}$. It is clear that the system consists of four distinct regions: bulk water, bulk organic and two interfaces. The bulk densities in the two-phase systems are in good agreement with values determined for the pure components using the same models $^{46}\left(1.000 \mathrm{~kg} / \mathrm{m}^{3}\right.$ for SPC/E water, $1.171 \mathrm{~kg} / \mathrm{m}^{3}$ for OPLS nitrobenzene and $1.160 \mathrm{~kg} / \mathrm{m}^{3}$ for MB nitrobenzene). The interfacial regions are characterized by a smooth transition in the density of both species from bulk values to zero. The water profile is extremely smooth, while the organic profile shows some density oscillations that persist inside the bulk region (more pronounced for the OPLS model).

\section{Figure 2}

In principle, there are two limiting cases that can produce a gradual decrease in density perpendicular to the interface: the interface is flat and the two phases intermix within a finite layer; or the interface is molecularly sharp but is broadened by thermal fluctuations. To verify which is the case for our system, we employ a procedure analogous to the one used by Linse ${ }^{16}$ to decouple density fluctuations in the $z$ direction from those in the $x y$ plane. We begin by dividing the simulation box in prisms of size $\frac{L_{\mathrm{x}}}{N} \times \frac{L_{\mathrm{y}}}{N} \times L_{\mathrm{z}}$ (i.e., the $x y$ plane is divided into a mesh of $N \times N$ squares). In each sub-box, we locate the limits of each phase $(l)$, for both interfaces. This is defined as the $z$ coordinate of the site of component $i$ that protrudes furthest into the opposite phase but is still linked to the bulk of phase $i$ (thus excluding overhangs and dissolved molecules from the definition of the interface). We have carefully verified that this criterion is robust and yields results that are reproducible. A diagram illustrating our method for determining the phase limits at two different values of $N$ is shown in Figure 3.

Figure 3 
Once the phase limits have been found, the interface positions $(h)$ and widths $(w)$ can be calculated according to:

$$
\begin{aligned}
& h_{\mathrm{L}}=\frac{l_{\mathrm{WL}}+l_{\mathrm{OL}}}{2} \\
& h_{\mathrm{R}}=\frac{l_{\mathrm{WR}}+l_{\mathrm{OR}}}{2} \\
& w_{\mathrm{L}}=l_{\mathrm{WL}}-l_{\mathrm{OL}} \\
& w_{\mathrm{R}}=l_{\mathrm{OR}}-l_{\mathrm{WR}}
\end{aligned}
$$

where the subscripts $L, R, W$ and $O$ are for the left interface, the right interface, the water phase and the organic phase, respectively. In previous works where this method was employed, the maximum value of $N$ was such that $L / N$ was larger than the bulk correlation length. This originates from the fact that the method was introduced by Weeks ${ }^{48}$ in his derivation of a capillary wave Hamiltonian. Indeed, capillary wave theory requires a lower cutoff for the thermal fluctuation wavelength, on the argument that a "capillary wave" is no longer meaningful beyond molecular dimensions (defined by $\xi$ ). Here, however, we go beyond this limit in an attempt to obtain further insight into the intrinsic structure of the interface. As we will see below, it is necessary to go well beyond the bulk correlation length in order to obtain meaningful intrinsic properties.

The probability distributions of the interface position and width as a function of $N$ are shown in Figure 4 for the MB model with $L=2.5 \mathrm{~nm}$. Similarly to what was observed in previous simulation studies, the signature is that of an interface that is relatively sharp on a molecular scale but is broadened by thermal fluctuations. As such, the average width decreases with $N$ while the position distributions become broader. If there were a gradual change in composition over a flat interface, the width and probability distributions would be independent of $N$. Another aspect worth noticing is related to the shape of the width distributions. These show an approximately 
Gaussian shape until about $N=7$, but develop a tail extending to negative widths beyond this value. We will see later that beyond this limit the interface position is no longer unequivocally defined, and the concept of interface width as calculated above loses meaning.

\section{Figure 4}

The original form of CWT assumed a step function intrinsic density profile. In other words, the interface would be perfectly flat in the absence of capillary waves ${ }^{20}$. The mean-field approach, on the other hand, assumes that the intrinsic profile is smooth, but neglects interfacial broadening by capillary waves ${ }^{49}$. Weeks ${ }^{48}$ combined these two approaches and suggested the existence of a relatively smooth intrinsic profile (with an associated intrinsic width) that is then subject to capillary wave broadening. Since then, efforts have been devoted towards obtaining the intrinsic density profile from computer simulations of interfaces ${ }^{8-11,30,50}$. Almost a decade ago, our group proposed using the method of dividing the $x y$ plane into a mesh, described above, to calculate this profile ${ }^{8}$. The profiles thus obtained showed density oscillations in the vicinity of the interface that were dampened as the bulk region was approached. However, as in the calculation of the interface width and position, the intrinsic profiles were computed at a resolution of $L / N \approx$ $\xi$, based on the arguments of CWT. However, as shown later by Tarazona and co-workers ${ }^{9-11}$, a correct intrinsic density profile can only be obtained by eliminating the fluctuations of the interface location even beyond the limits of applicability of CWT. In other words, one must use a lower wavelength cutoff for the fluctuations that is close to the value of the Lennard-Jones site diameter of the liquid ${ }^{10}$. The profile calculated in this way is free from thermal fluctuations of the interface position and shows the true intrinsic density variation at the interface. These ideas have prompted us to extend our method of calculation to cross-sectional areas well below the squared bulk correlation length. The resulting profiles for both water and MB nitrobenzene, with $L=2.5$ nm, are shown in Figure 5. 


\section{Figure 5}

At $N=1$, the profiles are relatively smooth since they are broadened by thermal fluctuations, as already discussed. As the effect of these fluctuations is eliminated by increasing $N$, the profiles develop more detail and two density peaks emerge near the interface. In the case of water, the height of the first peak increases until about $N=7$, after which the peak decreases in intensity. For nitrobenzene, the behavior is the same, but the limiting profile occurs at $N=8$. These values are not arbitrary, but make physical sense. For a given $N$, the water density profile is calculated relative to the limits of the organic phase (and vice-versa for the organic profile). These limits define a surface with a resolution of $L / N$. Naturally, this resolution cannot be smaller than the diameter of an atomic site $(\sigma)$, otherwise the location of the surface is not unambiguously defined. The largest nitrobenzene site is $0.355 \mathrm{~nm}$ in diameter ${ }^{46}$, which, for $L=$ $2.5 \mathrm{~nm}$, yields a limiting value of $N \approx 7$. This value of $N$ allows one to obtain a local definition of the surface of the organic phase, defined with atomic resolution. The intrinsic water density profile can then be calculated relative to this local definition. For the organic profile, we can take the diameter of the SPC/E oxygen site $(0.31656 \mathrm{~nm})$ and calculate a limiting value of $N \approx 8$. Thus, examining the evolution of the density profile with $N$ (Figure 5), we can see that they converge to the intrinsic profile at the limiting resolution and then dramatically change shape beyond this limit. Our analysis therefore validates the lower wavelength cutoff proposed by Tarazona and Chacón $^{10}$.

Figure 6 shows the water and nitrobenzene intrinsic profiles for both models and for different values of $L$ (for $L=3.5 \mathrm{~nm}$, the water intrinsic profile is obtained at $N=10$ and the organic intrinsic profile at $N=11$ ). The first conclusion to draw is that the intrinsic profiles are independent of system size, which means that our method of calculation gives reproducible results. Furthermore, the oscillations in the nitrobenzene profile extend further towards the bulk 
phase than in the case of water. It is also clear that the water intrinsic structure depends little on the specifics of the potential model used for nitrobenzene, since the curves for the OPLS and the MB models are practically indistinguishable. The nitrobenzene intrinsic profile, on the other hand, does depend on the potential model. As discussed by Chacón et al. ${ }^{50}$, the decay in oscillations in the intrinsic density profiles follow a behavior similar to the decay of oscillations in the bulk radial distribution function, reflecting the nature of molecular packing. This packing was shown to be slightly different for the two nitrobenzene models ${ }^{46}$. Tarazona and co-workers ${ }^{9}$ used a much more complex method for calculating the intrinsic profiles than the one proposed in this paper, based on using a set of pivot atoms to define the interface as a sum of Fourier components. The minimal-area intrinsic surface was refined until convergence by an iterative procedure. Their work focused originally on simple fluid models (such as the soft-alkali or the Lennard-Jones fluid) ${ }^{9-11}$ and was later extended to water ${ }^{50}$, but was applied only to liquid-vapor interfaces. In a recent paper, Chowdhary and Ladanyi ${ }^{30}$ have applied a slightly simplified version of Tarazona's method to water/alkane interfaces. They used a different algorithm for choosing the set of pivot atoms, and mapped all other sites to the surface atoms by applying a Voronoi tessellation operation. Despite these simplifications, their method is still much more laborious than our procedure. One conclusion drawn from that study was that the water intrinsic structure is not very affected by the nature of the organic phase. This is certainly consistent with our own observations regarding the small effect of the nitrobenzene model on the water profile. In light of this, it would be interesting to compare our water intrinsic profiles with those obtained by Chowdhary and Ladanyi. This is done in Figure 6a, where we can see that the water profiles are very similar. This not only supports claims that the water structure is virtually independent of the nature of the organic phase, but also validates our method for calculating the intrinsic profiles. We reinforce the idea that one must define the interfacial surface with a resolution close to the 
order of the characteristic site diameter so as to obtain meaningful profiles. Previous calculations where this was not verified ${ }^{8,26,27}$ do not represent the true intrinsic density profiles.

\section{Figure 6}

\subsection{Interfacial Tension and Width}

Perhaps the most important macroscopic property defining an interfacial system is the interfacial tension. This property can be calculated directly in molecular dynamics simulations using the so-called virial route, which relies on relating $\gamma$ to the integral of the components of the pressure tensor over the interfacial plane ${ }^{21}$. The inhomogeneities in pressure due to the interface produce a finite value of the tension. Using this method, the interfacial tension can be expressed for our system as:

$$
\gamma_{\mathrm{v}}=\frac{1}{2}\left\langle L_{\mathrm{z}}\left(P_{\mathrm{zz}}-\frac{P_{\mathrm{xx}}+P_{\mathrm{yy}}}{2}\right)\right\rangle
$$

where $P_{\mathrm{ij}}$ is the $i j$ component of the pressure tensor. The angle brackets denote an ensemble average, taking into account that in our simulations the box length in the direction normal to the interface is allowed to vary. The factor $1 / 2$ is due to the presence of two interfaces. Table 1 shows the calculated values of the interfacial tension from the virial route for our simulations. Compared to the experimental value for the water/nitrobenzene interface ${ }^{51}, 25.5 \mathrm{mN} / \mathrm{m}$, both the OPLS and the MB models overestimate the interfacial tension, with the latter yielding better agreement. The tension should depend strongly on the water/organic interactions, and in principle it should be possible to fine tune the values of the unlike pair interaction parameters to yield good agreement with experiment. However, such an exercise is beyond the scope of this paper.

\section{Table 1}

Another commonly used method to calculate the interfacial tension from simulations is by applying capillary wave theory. In this theory, $\gamma$ is related to the width of the interface induced by 
capillary wave fluctuations according to equation (1). Whether or not the computed values of $\gamma_{\mathrm{cw}}$ should agree with experiment will probably depend mostly on the quality of the potential models employed. However, if CWT is applicable, it is reasonable to expect good agreement between $\gamma_{\mathrm{cw}}$ and $\gamma_{N}$, since both calculations are performed for the same underlying model system. Benjamin ${ }^{17}$ determined $w_{\mathrm{cw}}$ from the mean squared deviation of the interface location distribution, calculated by dividing the interfacial plane into a square mesh of side $L / N=0.54 \mathrm{~nm}$. Using the width thus calculated and a value of $\xi=0.4 \mathrm{~nm}$ in equation (1), he obtained a result for $\gamma_{\mathrm{cw}}$ of the water/1,2dichloroethane interface that was significantly below the experimental value and the simulated $\mathcal{N}$ (which suffered from large statistical errors). Michael and Benjamin ${ }^{6}$ have applied equation (1) to the water/nitrobenzene interface, estimating $w_{\mathrm{cw}}$ as half the position of the peak in the width distribution and assuming $\xi=0.6 \mathrm{~nm}$. Their result for $\gamma_{\mathrm{cw}}(30 \mathrm{mN} / \mathrm{m})$ was relatively close to the experimental value, but once again underestimated the interfacial tension calculated from the pressure tensor $(35 \mathrm{mN} / \mathrm{m})$. In their study of the water/ $\mathrm{CO}_{2}$ interface, da Rocha et al. ${ }^{25}$ used the mean square deviation of the interface location calculated at $L / N=0.7 \mathrm{~nm}$ to estimate the capillary wave width and, assuming $\xi=0.9 \mathrm{~nm}$, obtained interfacial tensions in good agreement with $\gamma_{\text {. }}$. Thus, apart from the last study mentioned, agreement between the liquid/liquid interfacial tension calculated in the same system from the virial route and from CWT is not usually found. In our view, this is due to three distinct but related effects: i) the method for determining the interfacial width is not well established; ii) it is commonly assumed that the whole width of the interface is due to capillary wave fluctuations (and thus the total interfacial width is used directly in equation (1)); iii) the bulk correlation length used in equation (1) is somewhat ambiguously defined.

As discussed above, Weeks ${ }^{48}$ proposed that the global interfacial density profile results from capillary wave broadening superimposed onto an intrinsic density profile. Thus, the total 
interfacial width $\left(w_{\mathrm{t}}\right)$ may be defined as the squared sum of the capillary wave width and the intrinsic width $\left(w_{\mathrm{i}}\right)$ :

$$
w_{\mathrm{t}}^{2}=w_{\mathrm{i}}^{2}+w_{\mathrm{cw}}^{2}
$$

For the original CWT, with an intrinsic step function profile, the intrinsic width is zero and the total width is equal to $w_{\mathrm{cw}}$. Sides et al. ${ }^{13}$ have demonstrated that equation (7) is strictly correct when the squared total and intrinsic widths are calculated from the variance of the respective density profiles. This hybrid theory has been successfully applied, for example, to describe experimental measurements of the interfacial width ${ }^{15}$, and was more recently tested in computer simulations of liquid/liquid interfaces by Senapati and Berkowitz ${ }^{14}$. These authors also proposed a method for calculating the interfacial tension without requiring an assumption for the bulk correlation length. In practice, their procedure involves performing at least two simulations with boxes of different cross-sectional area. One can then apply equation (1) to each simulation, and combine both expressions to eliminate $\xi$ (assuming, naturally, that this quantity is intrinsic to the system and does not vary with the box size). The resulting equation for the interfacial tension is ${ }^{14}$ :

$$
\gamma_{\mathrm{cw}}=\frac{k_{\mathrm{B}} T}{2 \pi\left(w_{\mathrm{cw}, I}^{2}-w_{\mathrm{cw}, I I}^{2}\right)} \ln \left(\frac{L_{I}}{L_{I I}}\right)
$$

where subscripts $I$ and $I I$ denote each of the simulation runs.

These latest theoretical advances provide us with the necessary tools to circumvent the sources of error described above, and to obtain values of $\gamma_{\mathrm{cw}}$ in good agreement with $\gamma_{v}$. The first step is to distinguish the total width of the interface from the width due to capillary wave fluctuations alone, by applying equation (7). The concept of an intrinsic width is plausible, since we have already seen that the intrinsic density profiles show a fair degree of smoothing. The next step is to calculate the total and intrinsic widths from the variance of the global and intrinsic 
density profiles, respectively. Sides et al. ${ }^{13}$ have calculated $w_{\mathrm{t}}$ by fitting the global profile to a function of the type:

$$
\rho(z)=\frac{\rho_{\mathrm{B}}}{2}\left[1+\operatorname{erf}\left(\frac{z}{\sqrt{2} w_{e}}\right)\right]
$$

where $\rho(z)$ is the $z$-dependent density, $\rho_{\mathrm{B}}$ is the equilibrium bulk density and $w_{\mathrm{e}}$ is a fitting parameter that is strictly equivalent to the square root of the variance of the profile. However, equation (9) is not able to capture the density fluctuations present in the intrinsic profiles. Thus, we have modified it by superimposing a damped oscillatory term onto the error function form, following Toxvaerd and Stecki ${ }^{29}$. Thus, our fitting function becomes:

$$
\rho(z)=\frac{\rho_{\mathrm{B}}}{2}\left[1+\operatorname{erf}\left(\frac{z}{\sqrt{2} w_{e}}\right)\right]\left[1+A \exp (B z) \cos \left(\frac{2 \pi}{C} z\right)\right]
$$

where $w_{\mathrm{e}}, A, B$ and $C$ are fitting parameters. $A$ controls the amplitude of the oscillations, $B$ controls their decay rate and $C$ is the oscillation period. In all fits, we have opted to keep $\rho_{\mathrm{B}}$ fixed at the equilibrium value of the pure component densities in order to reduce the number of free parameters. The square root of the variance of a profile described by equation (10) is no longer equal to $w_{\mathrm{e}}$, but is given instead by:

$$
w_{o}=\sqrt{(1+A)} w_{e}
$$

Figure 6 shows the result of fitting equation (10) to the intrinsic profiles. The water profile is very well described by this function, which is able to capture both peaks of the profile. The fit to the organic profile is not as good, most likely due to the more complex structure of the nitrobenzene liquid. However, the fit is still adequate for our purposes, since it is able to describe well the increase in density from zero to the first peak, thus providing a good estimate of the intrinsic width. The widths obtained from applying equations (10) and (11) to fit the global $\left(w_{\mathrm{t}}\right)$ 
and the local $\left(w_{\mathrm{i}}\right)$ density profiles are given in Table 1 . The values shown are an average over both components and both interfaces.

In possession of both the total and the intrinsic widths, one can now calculate the capillary wave contribution to the width of the interface from equation (7) and apply equation (8) to extract the value of the interfacial tension. We have done this with the data from both our simulations of the MB model (at $L=2.5$ and $3.5 \mathrm{~nm}$ ), using the widths given in Table 1, and obtained a value of $32.5 \mathrm{mN} / \mathrm{m}$ for the interfacial tension. This result is in very good agreement with the value calculated by the virial route (see Table 2). We can go one step further and calculate a value for $\xi$ using equation (1) and $\gamma_{\mathrm{cw}}$. We obtain a value of $0.58 \mathrm{~nm}$ for the bulk correlation length of the water/MB system. Using the same method, Senapati and Berkowitz ${ }^{14}$ have obtained a value of $0.416 \mathrm{~nm}$ for the system water/ $\mathrm{CCl}_{4}$. Comparing the size of the carbon tetrachloride molecule with that of nitrobenzene, it is expected that the bulk correlation length of the latter be somewhat larger. The average molecular separation in the bulk organic liquid can be estimated by the position of the main peak of the RDF between the molecular centers of mass. For nitrobenzene, this value is around $0.65 \mathrm{~nm}^{46}$, and thus our result of $0.58 \mathrm{~nm}$ appears to be a reasonable estimate for $\xi$. Furthermore, this value should not be very sensitive to the model used for nitrobenzene, provided the molecular structure is similar. Thus, assuming $\xi=0.58 \mathrm{~nm}$ for the OPLS model, one can estimate the interfacial tension applying equation (1) again. The result, shown in Table 2, is once more in very good agreement with the computed $\gamma_{v}$.

\section{Table 2}

Using the above procedure, we obtain interfacial tensions that are not very sensitive to the particular function used to fit the density profiles, provided that the slope of the initial density increase is well described. Indeed, the results obtained using equation (9) are very close to those obtained when equation (10) is employed (see Table 2). Another possibility, used in several 
previous studies ${ }^{16,17,22,24-26}$, is to estimate the total interfacial width from the standard deviation of the distribution of interface positions (shown in Figure 4a). Following the reasoning described above, the correct value for the interfacial width should be calculated from the fluctuations in the location of the intrinsic interface (i.e., the distribution calculated by dividing the box length in segments of size $L / N \approx \sigma$ ). As such, we have calculated the total interfacial width by fitting the normalized intrinsic distributions of interface position to a Gaussian curve:

$$
P(l)=\frac{1}{w_{t} \sqrt{2 \pi}} \exp \left(-\frac{l^{2}}{2 w_{t}^{2}}\right)
$$

We then used these values of $w_{\mathrm{t}}$, also shown in Table 1 , together with the intrinsic widths calculated previously, in equations (8), (7) and (1) to obtain the interfacial tension and bulk correlation length, as described above. The results for $\gamma_{\mathrm{cw}}$ are not as close to $\gamma_{\mathrm{w}}$ as those obtained from fitting the density profiles, but the agreement is still very good. The value of $\xi$ is slightly more sensitive to the procedure employed but is still within an acceptable range.

\subsection{Radial Distribution Functions and Hydrogen Bonds}

So far, we have looked in detail at the properties of the interfacial region separating the water from the nitrobenzene phase. It is also interesting to examine to what extent these phases are affected by the presence of the interface. For that purpose, we have computed radial distribution functions in slices at different distances from the interface, as defined using the method described in section 3.1. Strictly speaking, the RDF in slice $i$ between a reference site $a$ and another site $b$ is built as a distribution of distances between all sites $b$ (in all slices) and each of the reference sites $a$ that have $z$ coordinates falling within the limits of slice $i$, normalized by the average number density of those sites in the bulk phase.

Figure 7 shows the RDFs between water oxygens $\left(\mathrm{O}_{\mathrm{W}}\right)$ and hydrogens $\left(\mathrm{H}_{\mathrm{W}}\right)$ for the water/MB system at $L=3.5 \mathrm{~nm}$, calculated using a global $(N=1)$ and a local $(N=10)$ definition of 
the interface. Looking first at the global profile (Figure 7a), the $\mathrm{O}_{\mathrm{W}}-\mathrm{H}_{\mathrm{W}} \mathrm{RDF}$ in the slice furthest away from the organic phase $(z=0.9 \mathrm{~nm})$ is identical to the one obtained in bulk SPC/E water ${ }^{43}$. As we move closer to the interface, there is a gradual depletion of the second peak, and the limiting value of the RDF also decreases. This is a direct consequence of the gradual decrease in water density as we move closer to the organic phase (see water density profile in Figure 5 for $N=1$ ). However, the position of the peaks and the intensity of the first peak (characteristic of hydrogen bonds) remain intact. This suggests that water keeps its highly H-bonded structure even in close vicinity to the organic phase. From the global profile, however, one cannot truly identify the water molecules that are in direct contact with nitrobenzene, since the position of this phase is smeared by thermal fluctuations. This can be achieved by calculating the intrinsic RDFs with a local definition of the limits of the organic phase (Figure $7 b$ ). In this case, we observe the same trend as in the local profile, except that the density depletion is much more pronounced (see water intrinsic profile in Figure 6). Even the water molecules that are protruding furthest into the nitrobenzene phase (lowest curve in Figure 7b) maintain the two-peak structure that is characteristic of the bulk phase (the peak intensity is much lower and the statistics are poorer due to the small number of water molecules in that slice). The RDFs computed in the water/OPLS system (not shown) are almost identical to those depicted in Figure 7, which reinforces the idea that the structure of the water phase is not strongly affected by the organic phase.

\section{Figure 7}

The $\mathrm{O}_{\mathrm{W}}-\mathrm{H}_{\mathrm{W}}$ RDFs of Figure 7 show that water retains its $\mathrm{H}$-bonded structure even beyond the interface location. This can be assessed in more detail by counting the number of hydrogen bonds formed between water molecules as a function of the distance to the interface. Two water molecules were considered to be hydrogen bonded if the distance between the hydrogen atom of one molecule and the oxygen atom of the other was below $0.24 \mathrm{~nm}$. The results of these calculations are shown in Figure 8. We can see that the number of H-bonds per molecule 
decreases as we move towards the organic phase (thin dashed line in Figure 8a). However, the water density is decreasing as well, and so is the number of molecules in the first coordination shell (thick dashed line). As a result of these two effects, the percentage of H-bonded water molecules actually increases from $73 \%$ in bulk to about $85 \%$ well beyond the global limit of the nitrobenzene phase. This observation has been made in previous simulations of liquid/liquid interfaces $^{6,16-18,22-27,31}$. However, we are now in a position to examine the intrinsic hydrogen-bond profile (Figure 8b). Once more, the same trend is observed, with a decreasing number of hydrogen bonds but with an increasing percentage of H-bonded molecules. In fact, practically all water molecules that are located well within the organic phase are hydrogen-bonded, which suggests that even these molecules show propensity to cluster together.

\section{Figure 8}

Figure 9 shows the RDFs between the carbon atoms of nitrobenzene that are connected to the nitro group $\left(\mathrm{C}_{\mathrm{N}}\right)$, as these are located practically at the centre of mass of the molecule. The curves furthest from the interface are identical to those of the pure component ${ }^{46}$. As expected for the global profile (Figure 9a), there is a gradual decrease in the intensity of the main peak and in the limiting value of the RDF, due to the decrease in nitrobenzene density as we approach the water phase. However, at a relative position of $z \approx 0 \mathrm{~nm}$ (i.e., close to the limit of the water phase), there is a change in the shape of the RDF: the shoulder at low $r$ (around $0.4 \mathrm{~nm}$ ) develops into a well-defined peak. This peak is characteristic of nitrobenzene molecules that are packed close together with a tendency for antiparallel alignment ${ }^{52}$. This change in shape is also evident in the local profile (Figure 9b), at a location that corresponds approximately to the first minimum of the intrinsic density profile. In other words, the presence of the interface induces closer packing and antiparallel alignment of the nitrobenzene molecules, and this effect extends significantly towards the bulk organic region. We will examine this packing effect in more detail when we study the molecular orientations in section 3.4. 


\section{Figure 9}

It is also worth examining the cross-species RDFs, i.e. those referring to interactions between water and organic sites. Figure 10 shows two such RDFs, namely that between water hydrogens and nitrobenzene oxygens $\left(\mathrm{O}_{\mathrm{N}}\right)$ and that between water oxygens and nitrobenzene hydrogens $\left(\mathrm{H}_{\mathrm{A}}\right)$. Previous simulations of water dissolved in nitrobenzene have identified these as the most important interactions occurring between those species ${ }^{53}$. It is clear that interfacial water interacts with nitrobenzene in much the same way as dissolved water. In fact, both interfacial RDFs are similar to their bulk counterparts, but with lower peak intensities and lower limiting values, due to the density depletion at the interface. In particular, it can be seen that water molecules at the interface establish hydrogen bonds via their hydrogen atoms with nitrobenzene oxygens, since both peaks characteristic of these bonds are present in the interfacial RDF. To quantify these interactions, we have plotted in Figure 8 the number of $\mathrm{H}_{\mathrm{W}}-\mathrm{O}_{\mathrm{N}}$ bonds per water molecule as a function of the distance to the interface (dotted lines). It is clear that this number increases as we approach the bulk nitrobenzene phase, partly compensating for the decrease in water-water bonds. In fact, water molecules protruding well into the organic phase establish a significant proportion of bonds with nitrobenzene molecules. As we will see below, these hydrogen bonds will have a pronounced effect on the orientation of the interfacial molecules.

\section{Figure 10}

\subsection{Molecular Orientation}

In this section we present results obtained using the method of Jedlovszky et al. ${ }^{12}$ for calculating the bivariate orientation distribution. However, we go one step further by calculating also this distribution relative to a local definition of the interface. Following Jedlovszky et al. ${ }^{12}$, the two chosen angles are: i) the angle between the interface normal and the water dipolar vector $(\theta)$; ii) the angle between the molecular normal vector and the projection of the interface normal onto the plane perpendicular to the dipolar vector $(\varphi)$. Due to the equivalence between two 
possible molecular normal vectors pointing in opposite directions, angle $\varphi$ falls in the range $0^{\circ} \leq \varphi \leq 90^{\circ}$. With this choice of vectors, a uniform orientation of water leads to uniform distributions of $\varphi$ and of $\cos (\theta)$. Details of this procedure and a diagram depicting the chosen angles and vectors may be seen in the original publication by Jedlosvsky et al. ${ }^{12}$.

The bivariate water distributions at different positions relative to the organic phase for the water/MB system at $L=3.5 \mathrm{~nm}$ using a global definition of the interface (i.e. for $N=1$ ) are shown in Figure 11. As expected, in the slices furthest from nitrobenzene water molecules adopt an isotropic orientation, typical of bulk water. As we move closer to the interface, they begin to align themselves with their molecular plane parallel to the interfacial plane $\left(\theta=90^{\circ} ; \varphi=0^{\circ}\right)$. Finally, the water molecules that are protruding into the organic phase have one hydrogen pointing into the nitrobenzene phase and their molecular plane is perpendicular to the interfacial plane $\left(\theta=30^{\circ} ; \varphi=90^{\circ}\right)$. These results are very similar to those obtained by Jedlovszky et al..$^{34}$, which once again confirms that the orientation of the water phase depends little on the nature of the organic phase.

\section{Figure 11}

We can now look at the same bivariate distributions, but based on a local definition of the interface (i.e. for $N=10$ ), which are plotted in Figure 12. Both the isotropic orientation, for bulk water, and the parallel orientation $\left(\theta=90^{\circ} ; \varphi=0^{\circ}\right)$, for interfacial water, are present in the intrinsic distributions. Interestingly though, the perpendicular orientation $\left(\theta=30^{\circ} ; \varphi=90^{\circ}\right)$ is reduced to a small ridge in the slices closest to the organic phase. This means that one cannot speak in terms of a "layer" of water molecules possessing this arrangement. Rather, it is only the molecules that belong to water "fingers" protruding well into the organic phase (which are almost completely surrounded by nitrobenzene molecules) that are oriented perpendicularly to the interface. When the effect of these corrugations is eliminated by adopting a local definition of the interface, the perpendicular arrangement is washed out by the much larger proportion of parallel 
water molecules. One can also observe that molecules showing a preference for parallel orientation relative to the interfacial plane are restricted to the first peak of the density profile. In other words, molecules that are also in contact with nitrobenzene but that are in close proximity to many other water molecules are aligned parallel to the interfacial plane, while molecules that are completely surrounded by other water molecules show a virtually bulk-like isotropic orientation. The same conclusions were drawn by Chacón et al. ${ }^{50}$ in their study of the intrinsic orientation of water at the liquid-vapor interface. These authors have observed that only water molecules at the interface align parallel to the interfacial plane, but that this orientation is correlated with the local curvature of the interface, such that molecules belonging to extrusions of the surface (with positive curvature) align perpendicularly to the interface, with one hydrogen pointing towards the vapor.

\section{Figure 12}

We have applied the method of bivariate distributions to nitrobenzene molecules as well, defining $\theta$ as the angle between the interface normal and the vector bisecting the nitro group (i.e. the vector that connects the nitrogen atom to the halfway point between the two $\mathrm{O}_{\mathrm{N}}$ atoms), and $\varphi$ as the angle between the vector normal to the nitro group and the projection of the interface normal onto the plane perpendicular to the bisector vector. This is analogous to the definition for water molecules, but we substitute the water oxygen by the nitrogen and the water hydrogens by the nitrobenzene oxygens. Strictly speaking, the orientation of the full nitrobenzene molecule cannot be uniquely defined by two vectors, since it can rotate along the $\mathrm{C}_{\mathrm{N}}-\mathrm{N}$ bond. However, previous simulations have shown that the nitro group is preferentially aligned with the aromatic plane and that the nitrobenzene molecule is quite rigid ${ }^{46}$. Furthermore, we are mostly interested in examining the orientation of the nitro group itself, since this is the group involved in hydrogen bonds with water ${ }^{53}$. 
The bivariate nitrobenzene orientation distributions for the water/MB system at $L=3.5 \mathrm{~nm}$ are shown in Figure 13 using a global definition of the interface and in Figure 14 with a local definition. The nitrobenzene molecules that are in contact with the water phase (first three slices in Figure 13 and second slice in Figure 14) are oriented with their plane parallel to the interface $\left(\theta=90^{\circ} ; \varphi=0^{\circ}\right)$. This preferential orientation was previously inferred from a monovariate distribution calculated on the same interface ${ }^{6}$, albeit with much more statistical noise. The intrinsic distributions also show that a small number of nitrobenzene molecules protruding far into the water phase (first slice in Figure 14) show a deviation from the parallel orientation and tilt their nitro groups toward the organic phase. As we move away from the water phase, the orientation shifts and the nitro groups become oriented with both the bisector and the normal vectors parallel to the interface $\left(\theta=90^{\circ} ; \varphi=90^{\circ}\right)$, i.e. with one of the oxygens pointing into the water phase and the other one toward the organic phase. These molecules are mostly in contact with other nitrobenzene molecules, but some of them may still be in contact with water. Moving further into the organic phase, there is a layer of nitrobenzene molecules oriented perpendicular to the interfacial plane with the bisector vector pointing either to the water phase $\left(\theta=0^{\circ}\right)$ or to the organic phase $\left(\theta=180^{\circ}\right)$. This layer is located approximately at the location where the $\mathrm{C}_{\mathrm{N}}-\mathrm{C}_{\mathrm{N}}$ RDF changes shape and develops a peak at close $r$ (see Figure 9), confirming that this peak is indeed due to nitrobenzene dimers packed in an antiparallel arrangement ${ }^{52}$. Beyond this layer, the distributions recover the isotropic orientation typical of the bulk liquid. It is worth noticing that the orientational anisotropy caused by the interface persists further into the bulk organic phase than into the water phase, in tune with the oscillations in the density profiles (see Figure 6). Results obtained in the water/OPLS system (not shown) are qualitatively very similar to those for the water/MB system.

\section{Figure 13}

\section{Figure 14}


Based on the analysis of our results, we can draw a picture of the interactions taking place at the water/nitrobenzene interface. Such as scheme is depicted in Figure 15. For the most part, both interfacial water and nitrobenzene molecules are oriented with their molecular plane parallel to the interface (molecules b, c and e in Figure 15). These interfacial layers consist of closely packed molecules and are responsible for the first density peaks shown in the intrinsic density profiles (see Figure 6). However, thermal fluctuations induce the appearance of corrugations, leading some molecules to appreciably penetrate the opposite phase. In the case of water, these molecules are oriented with one of the hydrogens pointing directly to the organic phase (molecule a in Figure 15). As discussed by Jedlovszky and co-workers ${ }^{12}$, this is due to the propensity of these protruding water molecules to form a hydrogen bond with one of the water molecules in the parallel layer (see dashed line between molecules a and b in Figure 15). However, in our system this orientation has a second advantage - it allows for the protruding water molecule to form a hydrogen bond with a nitrobenzene oxygen (dashed line between molecules a and d in Figure 15). We have already shown evidence in Figure 8 for the formation of these water/nitrobenzene bonds. This type of bond causes some of the nitrobenzene molecules to be oriented with one oxygen atom pointing into the water phase (molecule $d$ in Figure 15). As we move from the interfacial water layer into bulk water, the isotropic orientation and equilibrium density are recovered fairly quickly, due to the small molecular size of water and the high resilience of its hydrogen-bonded network. Nitrobenzene, however, is a larger and less symmetric molecule, and the influence of the interface extends further into the bulk region. Indeed, beyond the parallel interfacial layer, we still observe a thick layer of molecules that are predominantly perpendicular to the interface, with alternating directions of the dipole vector (see Figures 13 and 14).

\section{Figure 15}

\subsection{Self-diffusion}


We move now to an investigation of the dynamic properties of the interface, by looking at the diffusion coefficients of water and nitrobenzene. The diffusion coefficient in a given Cartesian direction (say, $x$ ) can be calculated from the molecular mean-square displacement in that direction, following Einstein's relation:

$$
\lim _{t \rightarrow \infty}\left\langle\left\|x_{i}(t)-x_{i}(0)\right\|^{2}\right\rangle=2 D_{x} t
$$

where $D_{\mathrm{x}}$ is the self-diffusion coefficient in the $x$ direction, $t$ is time and $x_{\mathrm{i}}(t)$ is the $x$ coordinate of particle $i$ at time $t$. In practice, the mean square displacement is calculated during a simulation run and plotted as a function of time. $D$ is obtained from the slope of the linear portion of the mean square displacement at long times. For statistical purposes, the diffusion coefficient is computed in each sampling block (see section 2), from which an average and standard deviation are calculated. We divide the simulation box in slices, as described in section 3.1 , and calculate $D$ in each slice in order to study the variation of the diffusion coefficient with distance to the interface, defined both globally and locally. In this work, we use slices that are $0.25 \mathrm{~nm}$ thick, much thinner than slices used in previous studies ${ }^{6,17,22-24,33}$, but we are able to obtain good statistics due to the long simulation times employed. The squared displacement of a molecule at time $t$ relative to the reference position (at time 0 ) is assigned to a given slice if the $z$ coordinate of that molecule at time $t$ falls within this slice. We have tested alternative assignment strategies (e.g. assigning the displacement to each slice according to the $z$ coordinate of the molecule at time 0 ) but found the results to be insensitive to this choice.

The results for the water diffusion coefficient in the water/MB system at $L=3.5 \mathrm{~nm}$ are shown in Figure 16. Since diffusion in the $x$ and $y$ directions is indistinguishable, only the average of these values is shown, and denoted $D_{\mathrm{xy}}$. This value (open symbols) tends toward the diffusion coefficient calculated in pure SPC/E water ${ }^{53}$, shown as the dashed line. As we move in the direction of the organic phase, the lateral diffusion coefficient shows a slight decrease. The 
diffusion coefficient perpendicular to the interface $\left(D_{z}\right)$, however, shows a remarkably different pattern. First of all, even well within the bulk phase, $D_{z}$ does not reach the pure-component value, which probably means that our box is still not long enough in the $z$ direction to eliminate all effects of the interface. Static properties, such as density profiles, hydrogen bonds and orientation distributions, are less sensitive to the presence of the interface and reach bulk values much earlier than dynamic properties like the diffusion coefficient. Moving towards the organic phase also brings about a decrease in $D_{\mathrm{z}}$. However, this property goes through a minimum, and molecules that protrude significantly into the organic phase are moving almost as fast as bulk molecules in the direction perpendicular to the interface. The minimum is present also in the local diffusion profile (Figure 16b), so it is intrinsic to the interface and not a consequence of corrugation effects.

\section{Figure 16}

The corresponding results for the nitrobenzene diffusion coefficient are shown in Figure 17. The trend observed for the lateral diffusion coefficient is the inverse of the water case, i.e. $D_{\text {xy }}$ increases slightly from the bulk value as we move toward the water phase. As for the perpendicular diffusion coefficient, we observe once more that it goes through a minimum as we move from bulk organic to water.

\section{Figure 17}

The general trends observed in Figures 16 and 17 can be understood if we think of two limiting cases for diffusion: i) bulk molecules, i.e., molecules completely surrounded by other molecules of the same species; ii) dissolved molecules, i.e., molecules completely surrounded by molecules of the opposite species. Clearly, as we move towards the bulk regions, the diffusion coefficients of both water and nitrobenzene tend toward case i), even though the perpendicular component does not reach the precise bulk value. When water is dissolved in nitrobenzene, its diffusion coefficient decreases substantially (for the MB model, is is $1.53 \times 10^{-9} \mathrm{~m}^{2} / \mathrm{s}$ ) ${ }^{53}$, due to 
restrictions in mobility imposed by the more viscous nitrobenzene liquid. Thus, both components of the water diffusion coefficient in Figure 16 should tend toward this value as we move into the bulk organic phase. Unfortunately, the statistics become very poor when we move into the nitrobenzene phase, due to the small number of dissolved water molecules. Nevertheless, it can be seen that $D_{\mathrm{xy}}$ and $D_{\mathrm{z}}$ tend to converge toward similar values, below the value for bulk water. In the case of nitrobenzene molecules, the opposite should happen - their diffusion should become faster when surrounded by less viscous and more mobile water molecules. Indeed, despite the poor statistics inside the water phase, this is the observed trend in Figure 17. These considerations are also able to explain some of the observed trends in previous studies ${ }^{6,17,22,24}$, although the statistical accuracy of the latter are generally poorer.

The limiting cases considered above explain the general trends, but are not sufficient to explain the minimum observed in $D_{z}$. Our results from the previous sections point to the existence of two interfacial layers, one on the water side and one on the organic side, with tightly packed molecules oriented parallel to the interfacial plane (see Figure 15). Most interfacial molecules prefer to reside in one of these regions, giving rise to a significant increase in density. The location of the minima in $D_{\mathrm{z}}$ roughly corresponds to the position of these layers. Thus, it can be said that these layers significantly reduce molecular mobility in the direction perpendicular to the interfacial plane, contributing towards the cohesion of individual phases. Occasionally, some of these molecules gather enough momentum to penetrate the opposite phase (through corrugations of the interface, caused by thermal fluctuations), and exhibit values of $D_{\mathrm{z}}$ that are similar to $D_{\mathrm{xy}}$. The fact that the minimum in $D_{\mathrm{z}}$ was not observed in previous studies ${ }^{6,17,22-24,33}$ is probably due to the poor statistics of the latter and/or to the fact that the system was divided in much thicker slices parallel to the interfacial plane.

\section{Conclusions}


In this paper we have presented results of a molecular dynamics simulation study of the water/nitrobenzene interface, using relatively large system sizes and long simulation runs. This allowed us to obtain results of significantly higher statistical accuracy than generally obtained in previous studies. More importantly, we have taken advantage of recent developments in theoretical descriptions of interfaces ${ }^{8-14}$, to obtain a detailed picture of the water/nitrobenzene interface. This systematic approach enabled us to clarify some inconsistencies present in the published literature on liquid/liquid interfaces.

Our results confirm that the water/nitrobenzene interface is relatively sharp on a molecular scale, but is substantially broadened by thermal fluctuations. These fluctuations induce a smoothing of the profiles calculated along the direction perpendicular to the interfacial plane. We have proposed a method for eliminating these fluctuations from the averaging procedure, thus obtaining true intrinsic density profiles. These show a relatively smooth increase in density followed by a layering effect, persisting to some degree into the bulk region (more pronounced in the case of nitrobenzene). The intrinsic profile for water is practically insensitive to the model used for nitrobenzene, and agrees very closely with profiles obtained by Chowdhary and Ladanyi $^{30}$ for water/hydrocarbon interfaces. By fitting our water and nitrobenzene profiles to an appropriate functional form, we were able to obtain an estimate of the intrinsic width of the interface. When combined with the total interfacial width (obtained either from a fit to the global density profiles or to the distribution of interface locations) this allows for the calculation of the width due to capillary wave fluctuations. Using capillary wave theory, this value can then be used to extract the interfacial tension and the bulk correlation length of the system. The interfacial tensions thus calculated agree very closely with values calculated directly from the simulations using the virial route. The bulk correlation lengths are also consistent with a definition based on molecular size. 
Using the proposed method for determining the interface surface, we are also able to obtain intrinsic profiles for several other properties of the system (radial distribution functions, hydrogen bonds, bivariate orientation distributions, diffusion coefficients). Our results point to the existence of two tightly packed interfacial layers, one on the water side and one on the organic side of the interface. Molecules in these layers tend to be oriented with their molecular planes parallel to the interfacial plane and show substantially restricted mobility in the direction perpendicular to the interface. Thus, they act as "buffer regions" aiming to preserve the integrity of the respective bulk phases. This strong interfacial layering may help explain recent X-ray reflectivity measurements of the water/nitrobenzene interface ${ }^{7}$.

Despite the presence of the interfacial layers, anisotropies imposed by the interface persist to some extent into the bulk regions. Dynamic properties, such as the diffusion coefficient, are the most strongly affected and, in our simulations, do not fully reach bulk values. Generally speaking, water is more effective at restoring bulk structure, due to its small molecular size and, perhaps more importantly, due to the high resilience of its hydrogen-bonded structure. Indeed, our results confirm previous conclusions that the structure of the water phase is only weakly affected by the nature of the organic phase. The structure of nitrobenzene is more sensitive to the presence of the interface and structural anisotropies persist much further into the bulk region. Molecules adjacent to the parallel layer in the direction of bulk orient with one oxygen pointing towards the water phase. This configuration is induced by hydrogen bonds formed between nitrobenzene oxygens and hydrogens belonging to water molecules that protrude farthest into the organic phase. Beyond this region, another layer of nitrobenzene molecules exists, consisting of closely packed molecules with an antiparallel dipolar arrangement. Only beyond this layer is isotropic bulk structure restored.

\section{Acknowledgments:}


The authors wish to acknowledge Dr. Rubin Gulaboski for helpful comments regarding electrochemical measurements at liquid/liquid interfaces. Thanks are also due to Fundação para a Ciência e Tecnologia (FCT), Lisbon, Portugal and to FEDER for financial support to REQUIMTE.

\section{References:}

[1] Benjamin, I. Annu. Rev. Phys. Chem. 1997, 48, 407.

[2] Marcus, Y. Ion Properties; Marcel-Dekker: New York, 1997.

[3] Scholz, F.; Schroeder, U.; Gulaboski, R. Electrochemistry of Immobilized Particles and Droplets; Springer: Heidelberg, Berlin, 2005.

[4] Gulaboski, R.; Mirceski, V.; Pereira, C. M.; Cordeiro, M. N. D. S.; Silva, A. F.; Quentel, F.; L'Her, M.; Lovric, M. Langmuir 2006, 22, 3404.

[5] Gulaboski, R.; Galland, A.; Bouchard, G.; Caban, K.; Kretschmer, A.; Carrupt, P. -A.; Girault, H. H.; Scholz, F. J. Phys. Chem. B 2004, 108, 4565.

[6] Michael, D.; Benjamin, I. J. Electroanal. Chem. 1998, 450, 335.

[7] Luo, G.; Malkova, S.; Pingali, S. V.; Schultz, D. G.; Lin, B.; Meron, M.; Benjamin, I.; Vanysek, P.; Schlossman, M. L. J. Phys. Chem. B 2006, 110, 4527.

[8] Fernandes, P. A.; Cordeiro, M. N. D. S.; Gomes, J. A. N. F. J. Phys. Chem. B 1999, 103, 8930.

[9] Chacón, E.; Tarazona, P. Phys. Rev. Lett. 2003, 91, 166103.

[10] Tarazona, P.; Chacón, E. Phys. Rev. B 2004, 70, 235407. 
[11] Chacón, E.; Tarazona, P. J. Phys.: Condens. Matter 2005, 17, S3493.

[12] Jedlovszky, P.; Vincze, A.; Horvai, G. J. Chem. Phys. 2002, 117, 2271.

[13] Sides, S. W.; Grest, G. S.; Lacasse, M.-D. Phys. Rev. E 1999, 60, 6708.

[14] Senapati, S.; Berkowitz, M. L. Phys. Rev. Lett. 2001, 87, 176101.

[15] Mitrinovic, D. M.; Tikhonov, A. M.; Li, M.; Huang, Z.; Schlossman, M. L. Phys. Rev. Lett. 2000, 85, 582.

[16] Linse, P. J. Chem. Phys. 1987, 86, 4177.

[17] Benjamin, I. J. Chem. Phys. 1992, 97, 1432.

[18] Carpenter, I. L.; Hehre, W. J. J. Phys. Chem. 1990, 94, 531.

[19] van Buuren, A. R.; Marrink, S.-J.; Berendsen, H. J. C. J. Phys. Chem. 1993, 97, 9206.

[20] Buff, F.; Lovett, R.; Stillinger, F. Phys. Rev. Lett. 1965, 15, 621.

[21] Rowlinson, J; Widom, B. Molecular Theory of Capillarity; Clarendon Press: Oxford, 1982.

[22] Michael, D.; Benjamin, I. J. Phys. Chem. 1995, 99, 1530.

[23] Schweighofer, K. J.; Benjamin, I. J. Electroanal. Chem. 1995, 391, 1.

[24] Fernandes, P. A.; Cordeiro, M. N. D. S.; Gomes, J. A. N. F. J. Phys. Chem. B 1999, 103, 6290.

[25] da Rocha, S. R. P.; Johnston, K. P.; Westacott, R. E.; Rossky, P. J. J. Phys. Chem. B 2001, $105,12092$. 
[26] Cordeiro, M. N. D. S. Mol. Simul. 2003, 29, 817.

[27] Chang, T.-M.; Dang, L. X. J. Chem. Phys. 1996, 104, 6772.

[28] Zhang, Y.; Feller, S. E.; Brooks, B. R.; Pastor, R. W. J. Chem. Phys. 1995, 103, 10252.

[29] Toxvaerd, S.; Stecki, J. J. Chem. Phys. 1995, 102, 7163.

[30] Chowdhary, J.; Ladanyi, B. M. J. Phys. Chem. B 2006, 110, 15442.

[31] Patel, H. A.; Nauman, E. B.; Garde, S. J. Chem. Phys. 2003, 119, 9199.

[32] Benjamin, I. J. Phys. Chem. B 2005, 109, 13711.

[33] Wang, H.; Carlson, E.; Henderson, D.; Rowley, R. L. Mol. Simul. 2003, 29, 777.

[34] Jedlovszky, P.; Vincze, A.; Horvai, G. J. Mol. Liq. 2004, 109, 99.

[35] Meyer, M.; Mareschal, M.; Hayoun, M. J. Chem. Phys. 1988, 89, 1067.

[36] Berendsen, H. J. C.; van der Spoel, D.; van Drunen, R. Comp. Phys. Comm. 1995, 91, 43.

[37] Lindahl, E.; Hess, B.; van der Spoel, D. J. Mol. Mod. 2001, 7, 306.

[38] Hockney, R. W.; Goel, S.P.J. J. Comput. Phys. 1974, 14, 148.

[39] Hess, B.; Bekker, H.; Berendsen, H. J. C.; Fraaije, J. G. E. M. J. Comp. Chem. 1997, 18, 1463.

[40] Nosé, S. Mol. Phys. 1984, 52, 255.

[41] Hoover, W. G. Phys. Rev. A 1985, 31, 1695.

[42] Parrinello, M.; Rahman, A. J. Appl. Phys. 1981, 52, 7182. 
[43] Berendsen, H. J. C.; Grigera, J. R.; Straatsma, T. P. J. Phys. Chem. 1997, 91, 6269.

[44] Jorgensen, W. L.; Tirado-Rives, J. J. Am. Chem. Soc. 1988, 110, 1657.

[45] Price, M. L. P.; Ostrovsky, D.; Jorgensen, W. L. J. Comput. Chem. 2001, 22, 1340.

[46] Jorge, M.; Gulaboski, R.; Pereira, C. M.; Cordeiro, M. N. D. S. J. Phys. Chem. B 2006, $110,12530$.

[47] Essman, U.; Perela, L.; Berkowitz, M. L.; Darden, T.; Lee, H.; Pedersen, L. G. J. Chem. Phys. 1995, 103, 8577.

[48] Weeks, J. D. J. Chem. Phys. 1977, 67, 3106.

[49] Fisk, S.; Widom, B. J. Chem. Phys. 1969, 50, 3219.

[50] Chacón, E.; Tarazona, P.; Alejandre, J. J. Chem. Phys. 2006, 125, 014709.

[51] Markin, V. S.; Volkov, A. G.; Volkova-Gugeshashvili, M. I. J. Phys. Chem. B 2005, 109, 16444.

[52] Janssen, R. H. C.; Theodorou, D. N.; Raptis, S.; Papadopoulos, M.G. J. Chem. Phys. 1999, $111,9711$.

[53] Jorge, M.; Gulaboski, R.; Pereira, C. M.; Cordeiro M. N. D. S. Mol. Phys. 2006, 104, 3627. 
Table 1 - Interfacial tensions and widths.

\begin{tabular}{l|lllll} 
system & $L(\mathrm{~nm})$ & $\gamma_{V}(\mathrm{mN} / \mathrm{m})$ & $w_{\mathrm{t}}(\mathrm{nm})^{\mathrm{a}}$ & $w_{\mathrm{i}}(\mathrm{nm})^{\mathrm{a}}$ & $w_{\mathrm{t}}(\mathrm{nm})^{\mathrm{b}}$ \\
\hline water/OPLS & 3.5 & $39.7 \pm 3.3$ & $0.1785 \pm 0.0043$ & $0.0487 \pm 0.0005$ & $0.1907 \pm 0.0078$ \\
water/MB & 3.5 & $32.4 \pm 3.2$ & $0.1973 \pm 0.0081$ & $0.0518 \pm 0.0009$ & $0.2056 \pm 0.0102$ \\
water/MB & 2.5 & $31.7 \pm 4.6$ & $0.1796 \pm 0.0107$ & $0.0529 \pm 0.0005$ & $0.1890 \pm 0.0130$ \\
\multicolumn{7}{c}{ a - Calculated from a fit to the density profiles. } \\
b - Calculated from a fit to the distributions of interface positions.
\end{tabular}

Table 2 - Interfacial tensions (in $\mathrm{mN} / \mathrm{m}$ ) and bulk correlation lengths (in $\mathrm{nm}$ ) calculated by different methods.

\begin{tabular}{l|l|l|ll|ll|ll|} 
system & $\gamma_{\exp }$ & $\gamma_{\mathrm{v}}$ & $\gamma_{\mathrm{cw}}{ }^{\mathrm{a}}$ & $\xi^{\mathrm{a}}$ & $\gamma_{\mathrm{cw}}{ }^{\mathrm{b}}$ & $\xi^{\mathrm{b}}$ & $\gamma_{\mathrm{cw}}{ }^{\mathrm{c}}$ & $\xi^{\mathrm{c}}$ \\
\hline water/MB & 25.5 & 32.1 & 32.5 & 0.58 & 32.6 & 0.55 & 33.0 & 0.48 \\
water/OPLS & 25.5 & 39.7 & 39.9 & 0.58 & 39.7 & 0.55 & 38.4 & 0.48
\end{tabular}

a - Calculated from a fit of the density profiles to equation (10).

${ }^{b}$ - Calculated from a fit of the density profiles to equation (9).

a - Calculated from a fit of the interface position distributions to equation (12). 
Figure 1. Snapshot of a simulation of the water/nitrobenzene interface showing the shape of the simulation box and the coordinate axes. Oxygen atoms are shown in blue, carbon atoms in purple, nitrogen atoms in green and hydrogen atoms in white.

Figure 2. Global density profiles for the water/OPLS (thin line) and water/MB (thick line). The organic phase is in the center and the water phase on both sides (see Figure 1).

Figure 3. Diagram illustrating, in two dimensions, our method for determining the limits of each phase at $N=1$ (left box) and $N=4$ (right box). The thick line represents the interface and the vertical lines represent the limits of the water (dotted) and organic (dashed) phases. The thin horizontal lines in the box on the right-hand side show the division of the interface in 4 subsections.

Figure 4. Probability distributions of the interface position (a) and width (b) for the system water $/ \mathrm{MB}$ at $L=2.5 \mathrm{~nm}$ at different mesh sizes dividing the $x y$ plane. The distributions are averaged over both interfaces.

Figure 5. Density profiles for the system water/MB at $L=2.5 \mathrm{~nm}$ at several sizes of mesh dividing the $x y$ plane. The profiles are averaged over both interfaces and, for clarity, are shifted in the $z$ direction so that their inflection point is at the origin.

Figure 6. Intrinsic density profiles for water (a) and nitrobenzene (b) relative to the limit of the opposite phase. The values of $N$ for each profile are as follows: water at $L=2.5 \mathrm{~nm}-N=7$; water at $L=3.5 \mathrm{~nm}-N=10$; nitrobenzene at $L=2.5 \mathrm{~nm}-N=8$; nitrobenzene at $L=3.5 \mathrm{~nm}-N=11$. The thick lines are fits to the OPLS profiles using equation (9) and the dashed-dotted line is drawn with data read from Ref. 21. 
Figure 7. $\mathrm{O}_{\mathrm{W}}-\mathrm{H}_{\mathrm{W}}$ radial distribution functions for the system water/MB at $L=3.5 \mathrm{~nm}$ in slices perpendicular to the interface, defined globally (a) and locally (b). The curves, from top to bottom, correspond to positions relative to the limit of the organic phase of: $0.9,0.7,0.5,0.3,0.1$, $-0.1,-0.3,-0.5,-0.7$ and -0.9 (negative values are within the organic phase).

Figure 8. Hydrogen bond profile as a function of position relative to the limit of the organic phase for the system water/MB at $L=3.5 \mathrm{~nm}$ using a global (a) and a local (b) definition of the interface. The thick dashed line is the number of molecules in the first coordination shell, the thin dashed line is the number of water-water hydrogen bonds per molecule, the full line is the percentage of bonded water molecules and the dotted line is the number of water-nitrobenzene hydrogen bonds per water molecule. The water density profiles (thick lines) are superimposed for ease of visualization.

Figure 9. $\mathrm{C}_{\mathrm{N}}-\mathrm{C}_{\mathrm{N}}$ RDFs for the system water/MB at $L=3.5 \mathrm{~nm}$ in slices perpendicular to the interface, defined globally (a) and locally (b). The curves correspond to the same positions relative to the limit of the water phase as in Figure 7, but the last five curves of part b are not statistically meaningful. The RDFs showing the onset of a peak at short distances are highlighted with thick lines.

Figure 10. $\mathrm{H}_{\mathrm{W}}-\mathrm{O}_{\mathrm{N}}$ (a) and $\mathrm{O}_{\mathrm{W}}-\mathrm{H}_{\mathrm{A}}$ (b) $\mathrm{RDF}$ for the system water/MB at $L=3.5 \mathrm{~nm}$ in the interface and in bulk.

Figure 11. Bivariate orientation distribution of water molecules for the system water/MB at $L=3.5 \mathrm{~nm}$ calculated in slices perpendicular to the interface using a global definition $(N=1)$. Red corresponds to high normalized probability and blue to low probability. Slices, from top to bottom, correspond to positions relative to the limit of the organic phase of: $-0.9,-0.7,-0.5,-0.3,-$ $0.1,0.1,0.3,0.5$ and 0.7 . 
Figure 12. Bivariate orientation distribution of water molecules for the system water/MB at $L=3.5 \mathrm{~nm}$ calculated in slices perpendicular to the interface using a local definition $(N=10)$. Red corresponds to high normalized probability and blue to low probability. Slices, from top to bottom, correspond to positions relative to the limit of the organic phase of: $-0.3,-0.1,0.1,0.3$, 0.5 and 0.7 (slices closer to the organic phase are not statistically meaningful).

Figure 13. Bivariate orientation distribution of nitrobenzene molecules for the system water/MB at $L=3.5 \mathrm{~nm}$ calculated in slices perpendicular to the interface using a global definition $(N=1)$. Red corresponds to high normalized probability and blue to low probability. Slices, from top to bottom, correspond to positions relative to the limit of the organic phase of: $-0.9,-0.7,-0.5,-0.3,-$ $0.1,0.1,0.3,0.5$ and 0.7 .

Figure 14. Bivariate orientation distribution of nitrobenzene molecules for the system water/MB at $L=3.5 \mathrm{~nm}$ calculated in slices perpendicular to the interface using a local definition $(N=11)$. Red corresponds to high normalized probability and blue to low probability. Slices, from top to bottom, correspond to positions relative to the limit of the organic phase of: $-0.1,0.1,0.3,0.5,0.7$ and 0.9 (slices closer to the water phase are not statistically meaningful).

Figure 15. Schematic representation of the structure of the water/nitrobenzene interface. The diagram shows a hypothetical cross section through the $y z$ plane (i.e., perpendicular to the interfacial plane). Color coding is the same as in Figure 1. The thick line represents the interface and the thin dashed lines represent hydrogen bonds.

Figure 16. Water diffusion coefficient for the system water/MB at $L=3.5 \mathrm{~nm}$ calculated in slices perpendicular to the interface, defined globally (a) and locally (b). Open symbols are for diffusion in the plane of the interface, closed symbols are for diffusion perpendicular to the 
interface and the dashed line is the pure-component diffusion coefficient. The density profiles (thick lines) are superimposed for ease of visualization.

Figure 17. Nitrobenzene diffusion coefficient for the system water/MB at $L=3.5 \mathrm{~nm}$ calculated in slices perpendicular to the interface, defined globally (a) and locally (b). Open symbols are for diffusion in the plane of the interface, closed symbols are for diffusion perpendicular to the interface and the dashed line is the pure-component diffusion coefficient. The density profiles (thick lines) are superimposed for ease of visualization. 


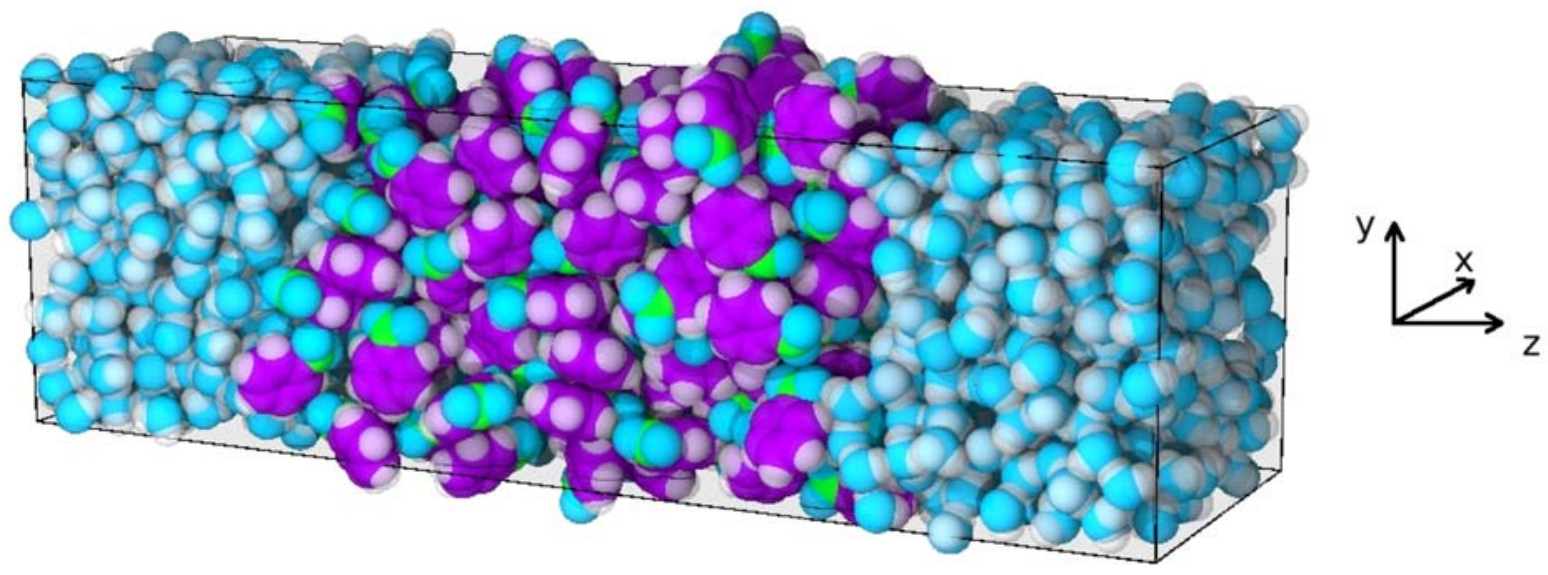

Figure 1. Snapshot of a simulation of the water/nitrobenzene interface showing the shape of the simulation box and the coordinate axes. Oxygen atoms are shown in blue, carbon atoms in purple, nitrogen atoms in green and hydrogen atoms in white. 


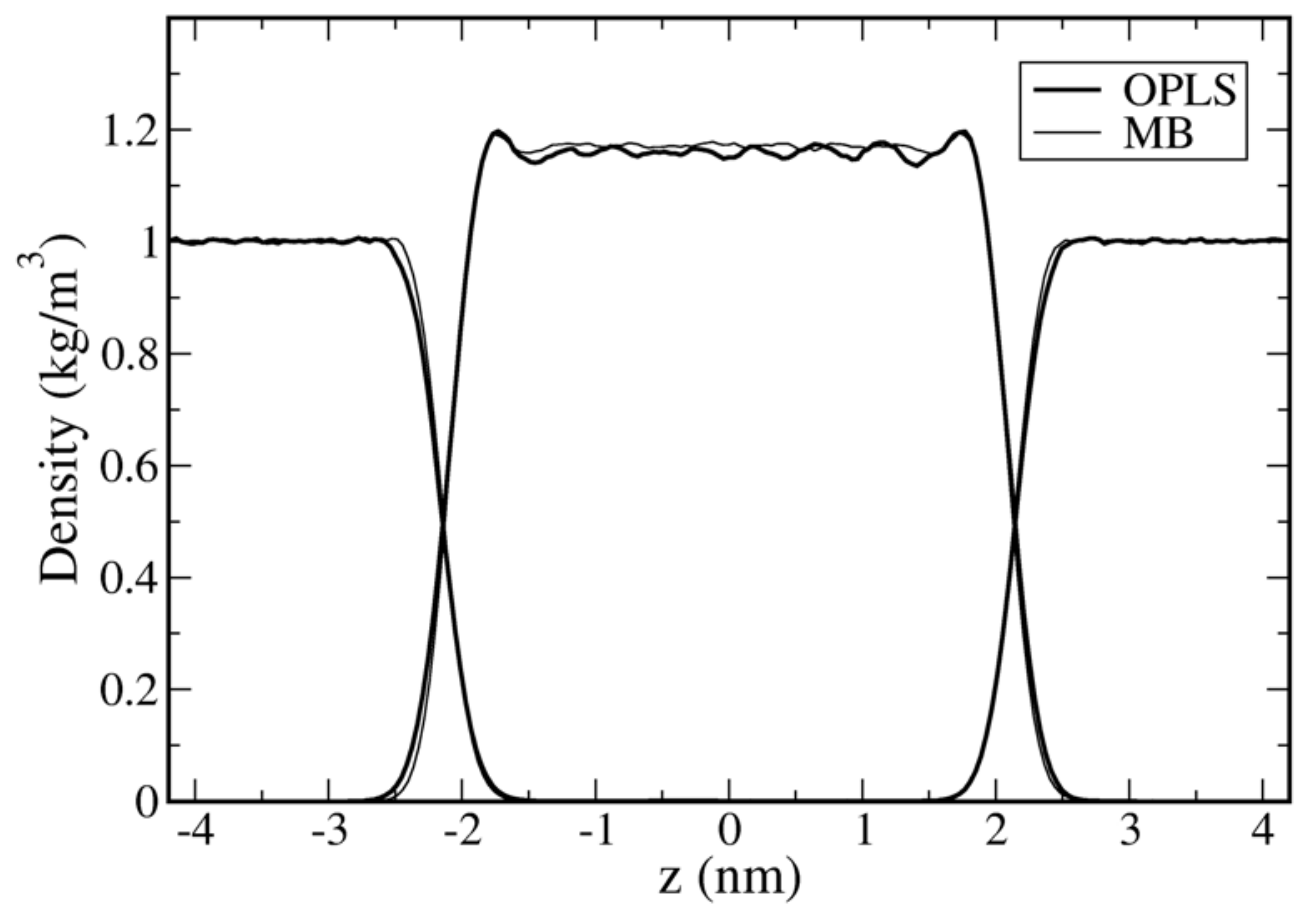

Figure 2. Global density profiles for the water/OPLS (thin line) and water/MB (thick line). The organic phase is in the center and the water phase on both sides (see Figure 1). 

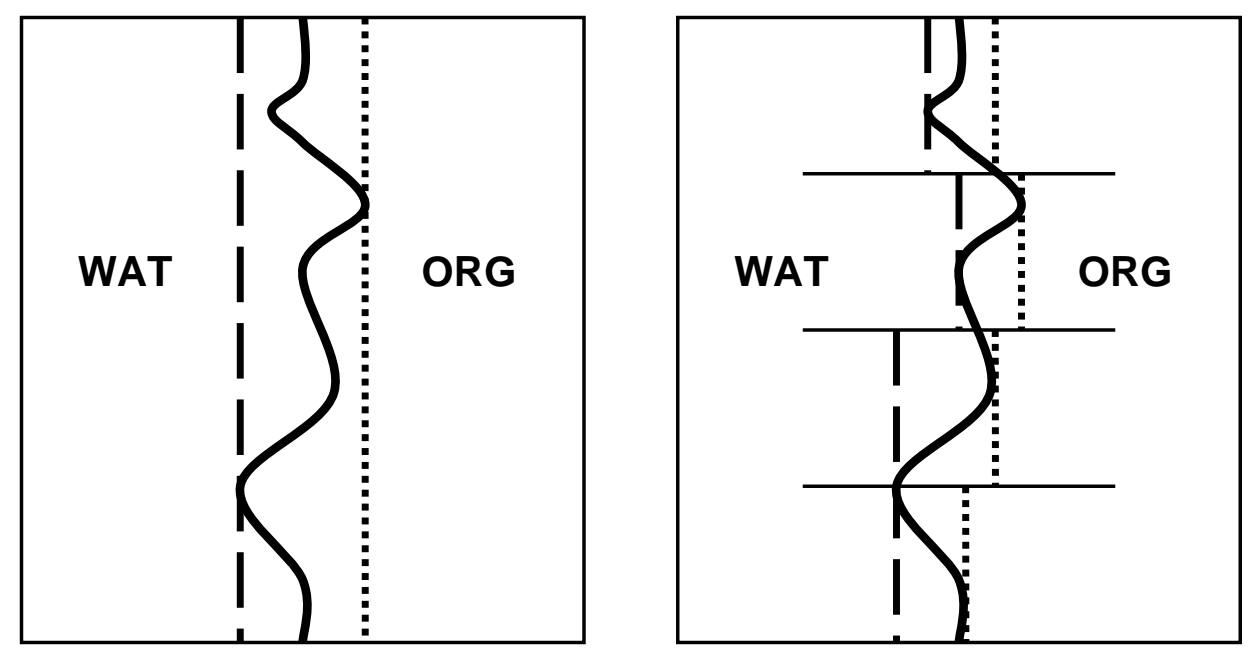

Figure 3. Diagram illustrating, in two dimensions, our method for determining the limits of each phase at $N=1$ (left box) and $N=4$ (right box). The thick line represents the interface and the vertical lines represent the limits of the water (dotted) and organic (dashed) phases. The thin horizontal lines in the box on the right-hand side show the division of the interface in 4 subsections. 

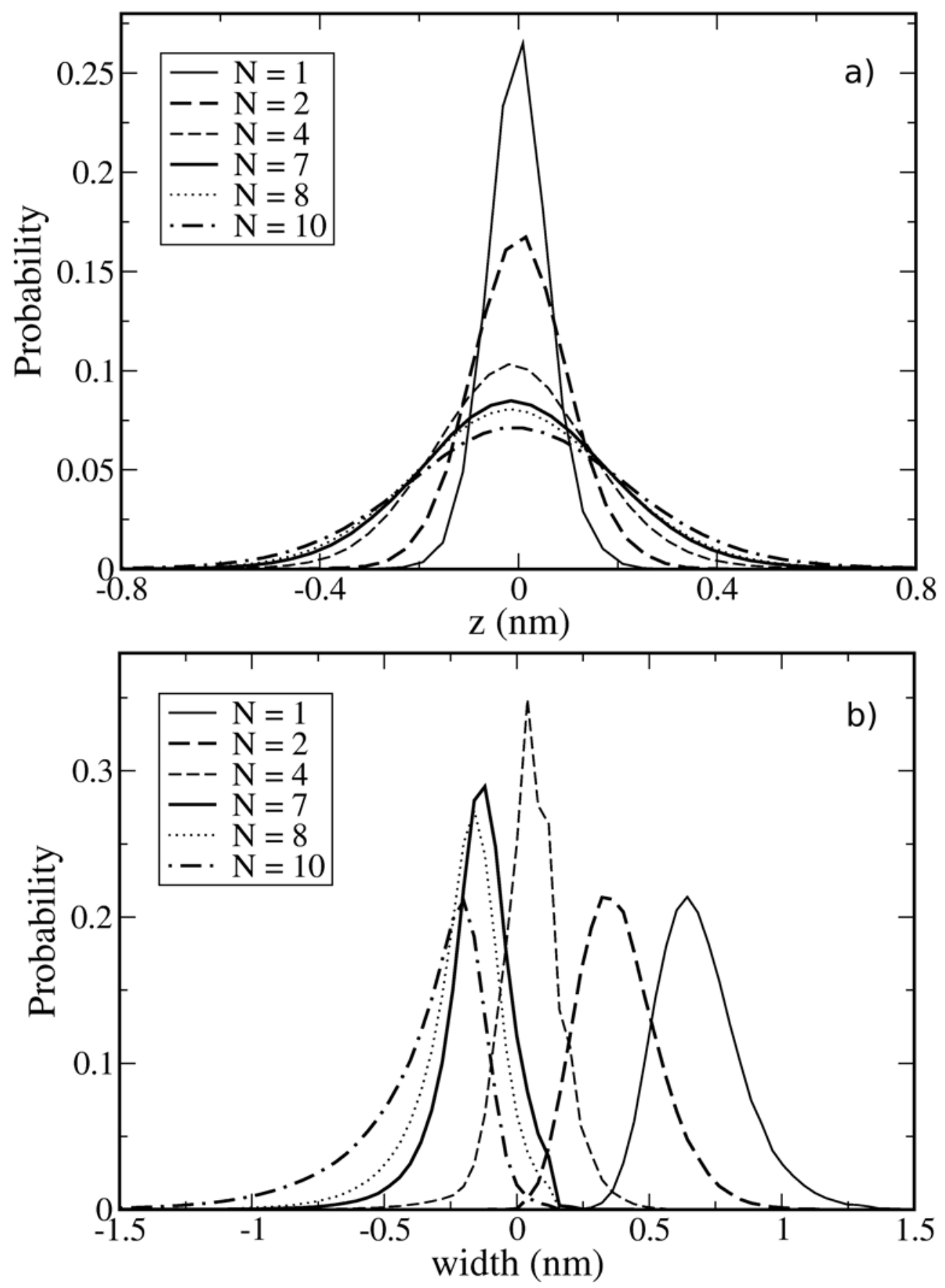

Figure 4. Probability distributions of the interface position (a) and width (b) for the system water/MB at $L=2.5 \mathrm{~nm}$ at different mesh sizes dividing the $x y$ plane. The distributions are averaged over both interfaces. 


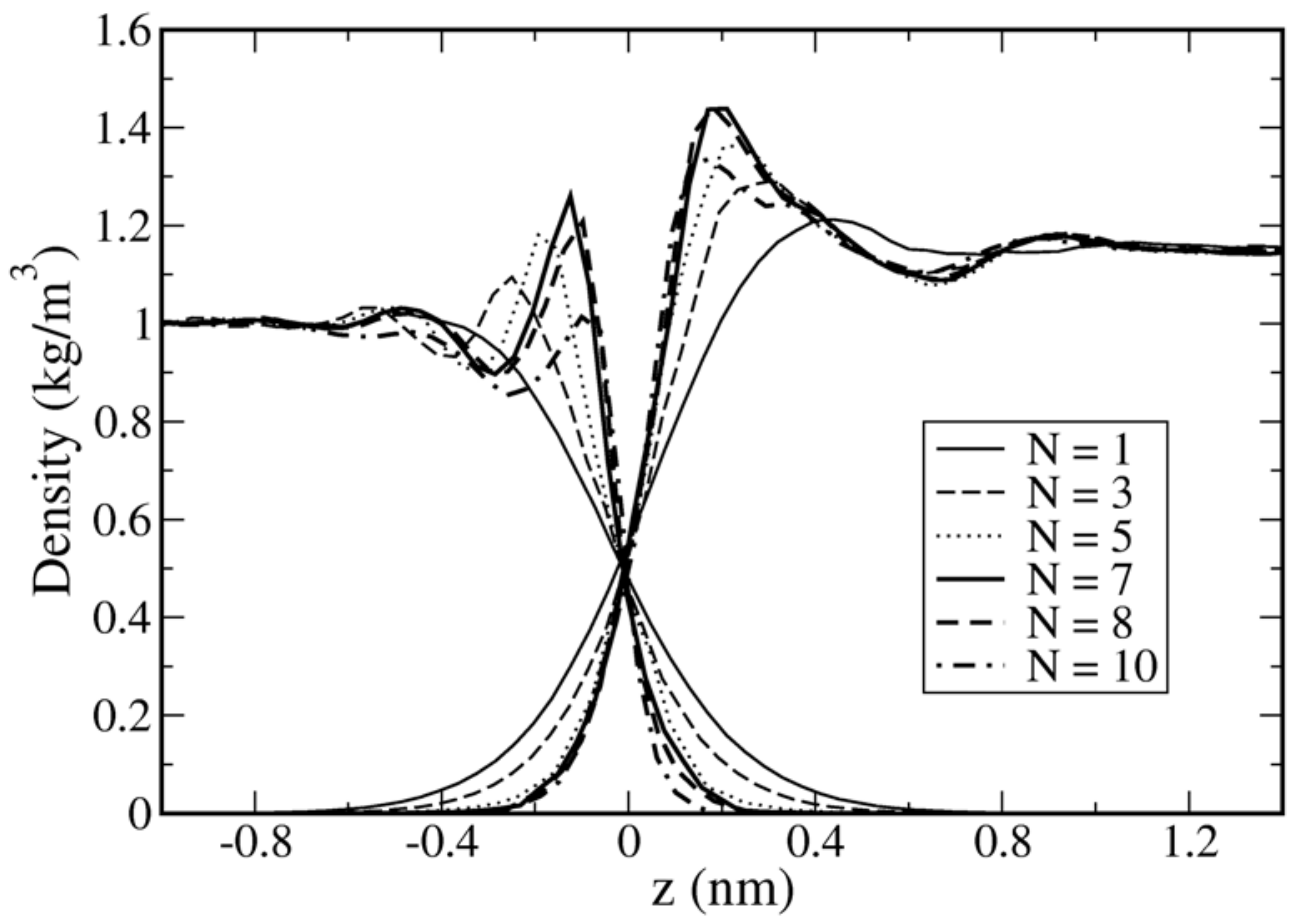

Figure 5. Density profiles for the system water/MB at $L=2.5 \mathrm{~nm}$ at several sizes of mesh dividing the $x y$ plane. The profiles are averaged over both interfaces and, for clarity, are shifted in the $z$ direction so that their inflection point is at the origin. 

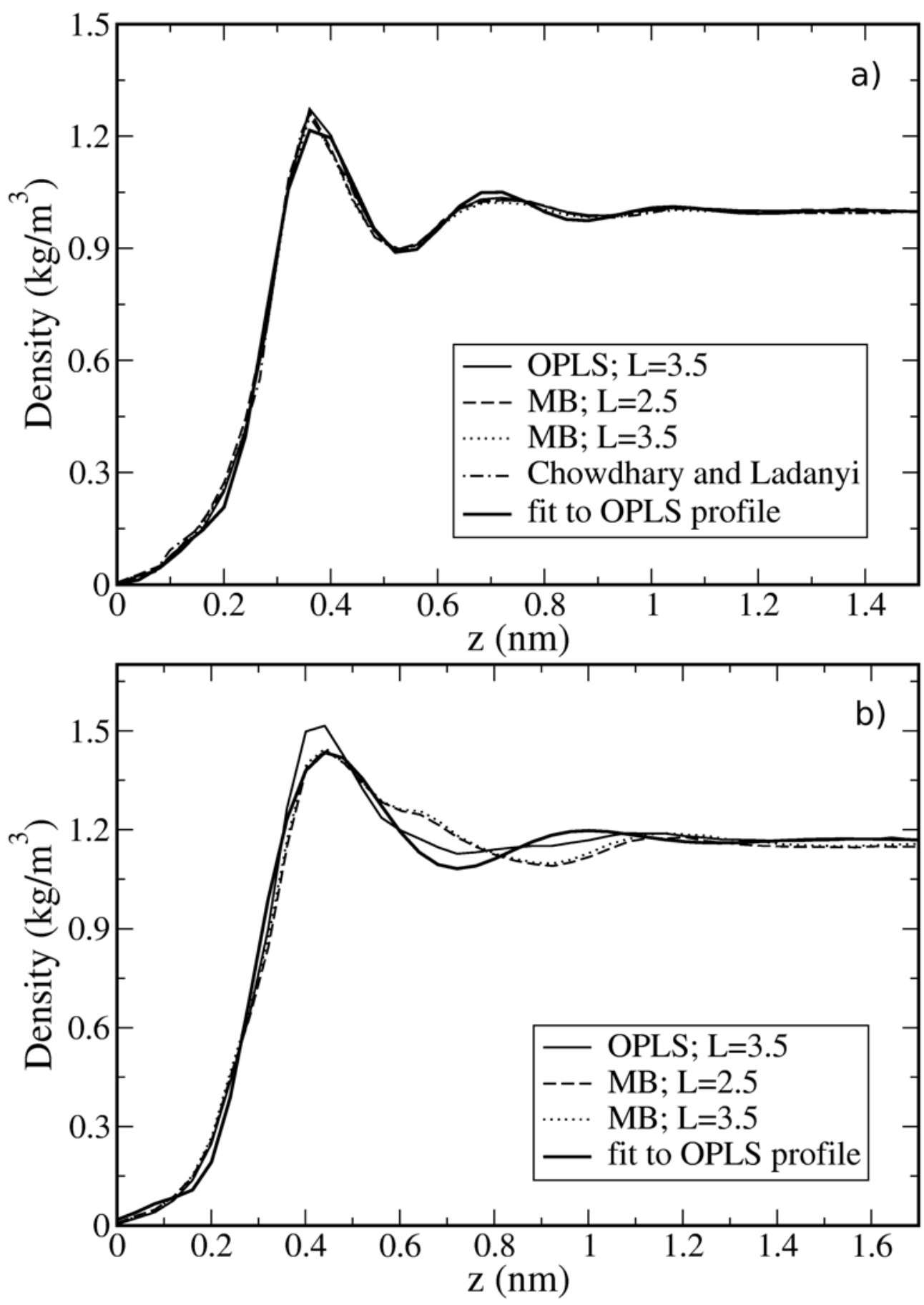

Figure 6. Intrinsic density profiles for water (a) and nitrobenzene (b) relative to the limit of the opposite phase. The values of $N$ for each profile are as follows: water at $L=2.5 \mathrm{~nm}-N=7$; water at $L=3.5 \mathrm{~nm}-N=10$; nitrobenzene at $L=2.5 \mathrm{~nm}-N=8$; nitrobenzene at $L=3.5 \mathrm{~nm}-N=11$. The thick lines are fits to the OPLS profiles using equation (9) and the dashed-dotted line is drawn with data read from Ref. 21. 

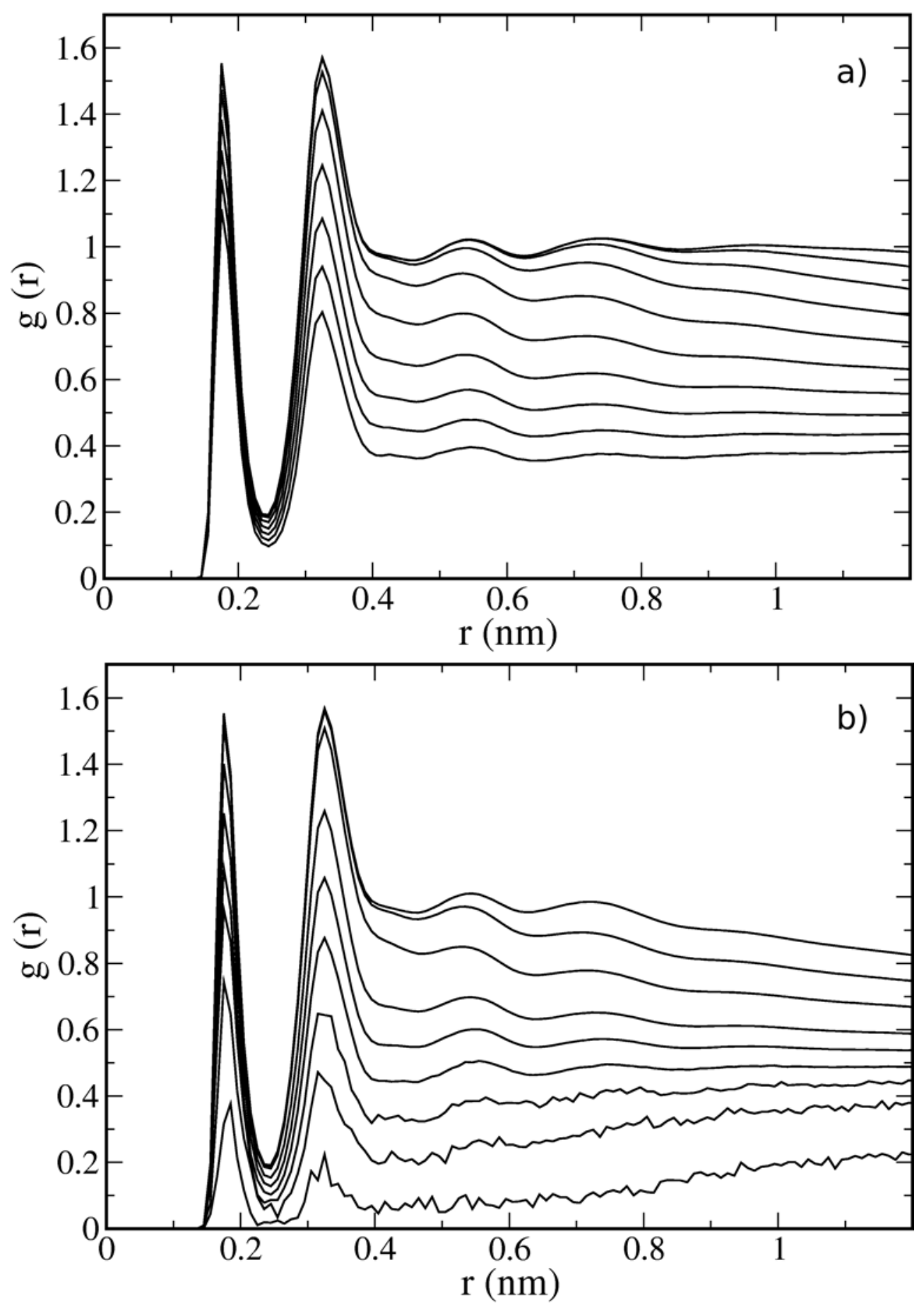

Figure 7. $\mathrm{O}_{\mathrm{W}}-\mathrm{H}_{\mathrm{W}}$ radial distribution functions for the system water/MB at $L=3.5 \mathrm{~nm}$ in slices perpendicular to the interface, defined globally (a) and locally (b). The curves, from top to bottom, correspond to positions relative to the limit of the organic phase of: $0.9,0.7,0.5,0.3,0.1$, $-0.1,-0.3,-0.5,-0.7$ and -0.9 (negative values are within the organic phase). 

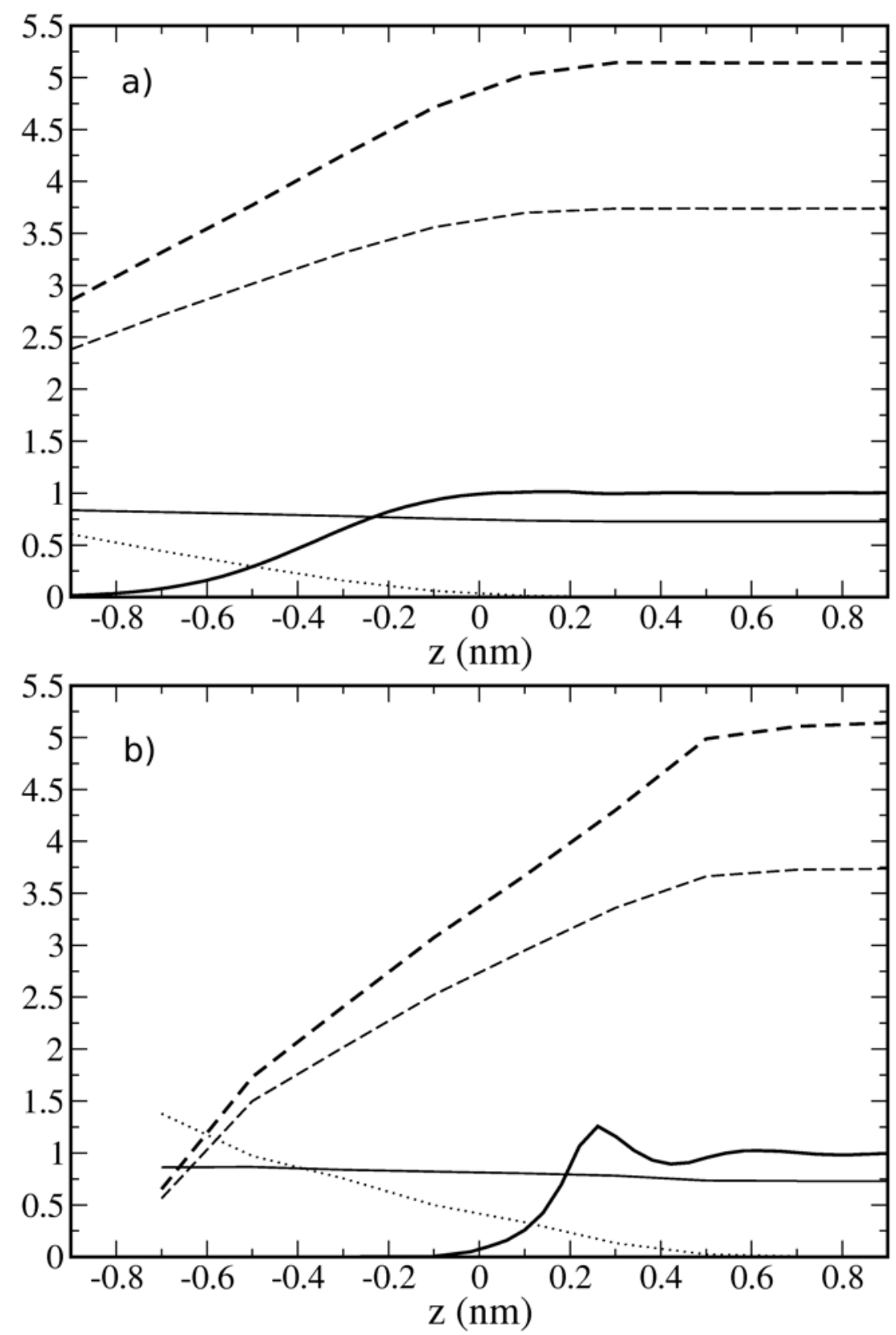

Figure 8. Hydrogen bond profile as a function of position relative to the limit of the organic phase for the system water/MB at $L=3.5 \mathrm{~nm}$ using a global (a) and a local (b) definition of the interface. The thick dashed line is the number of molecules in the first coordination shell, the thin dashed line is the number of water-water hydrogen bonds per molecule, the full line is the percentage of bonded water molecules and the dotted line is the number of water-nitrobenzene hydrogen bonds per water molecule. The water density profiles (thick lines) are superimposed for ease of visualization. 

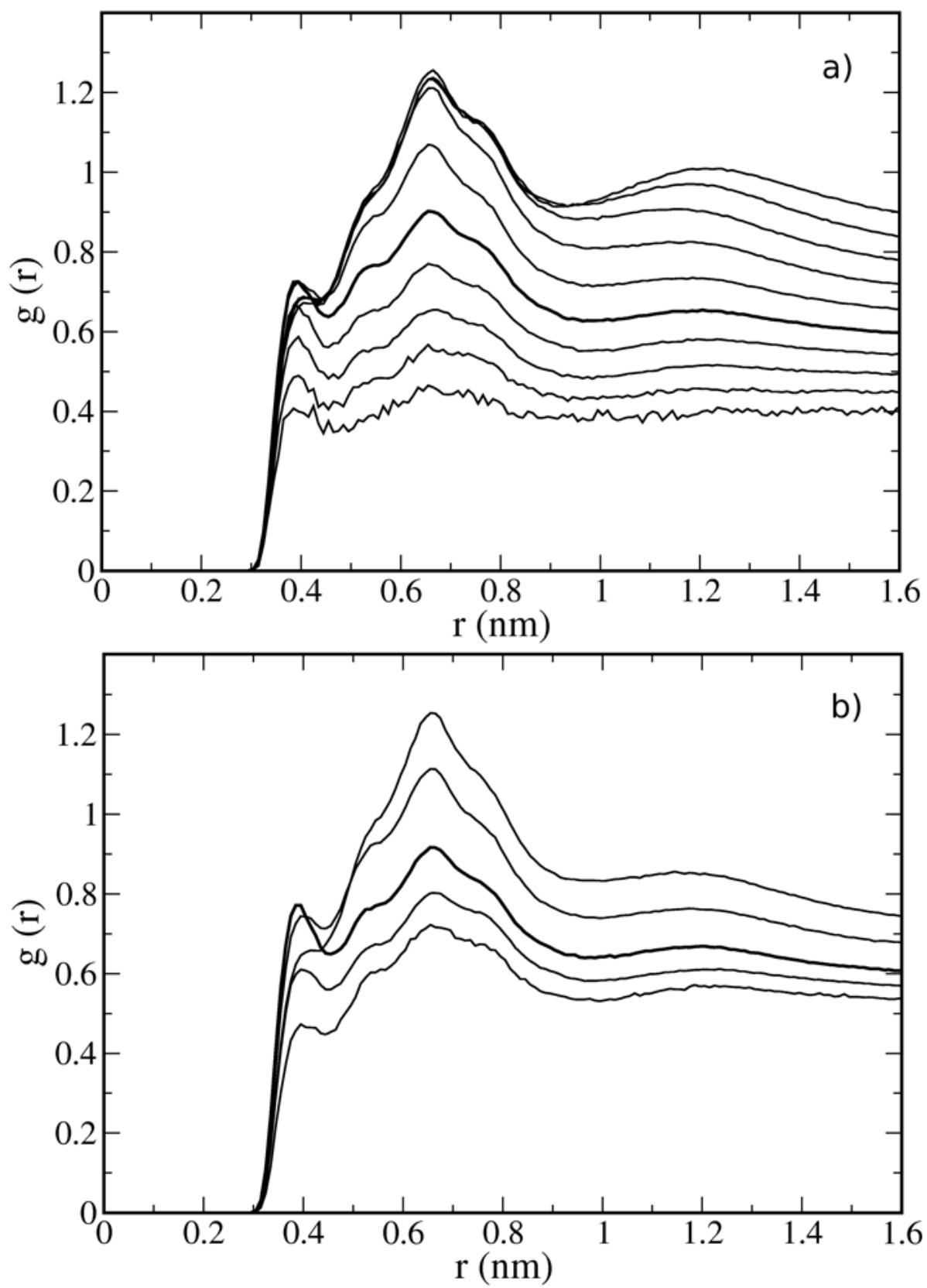

Figure 9. $\mathrm{C}_{\mathrm{N}}-\mathrm{C}_{\mathrm{N}} \mathrm{RDF}$ for the system water/MB at $L=3.5 \mathrm{~nm}$ in slices perpendicular to the interface, defined globally (a) and locally (b). The curves correspond to the same positions relative to the limit of the water phase as in Figure 7, but the last five curves of part b are not statistically meaningful. The RDFs showing the onset of a peak at short distances are highlighted with thick lines. 

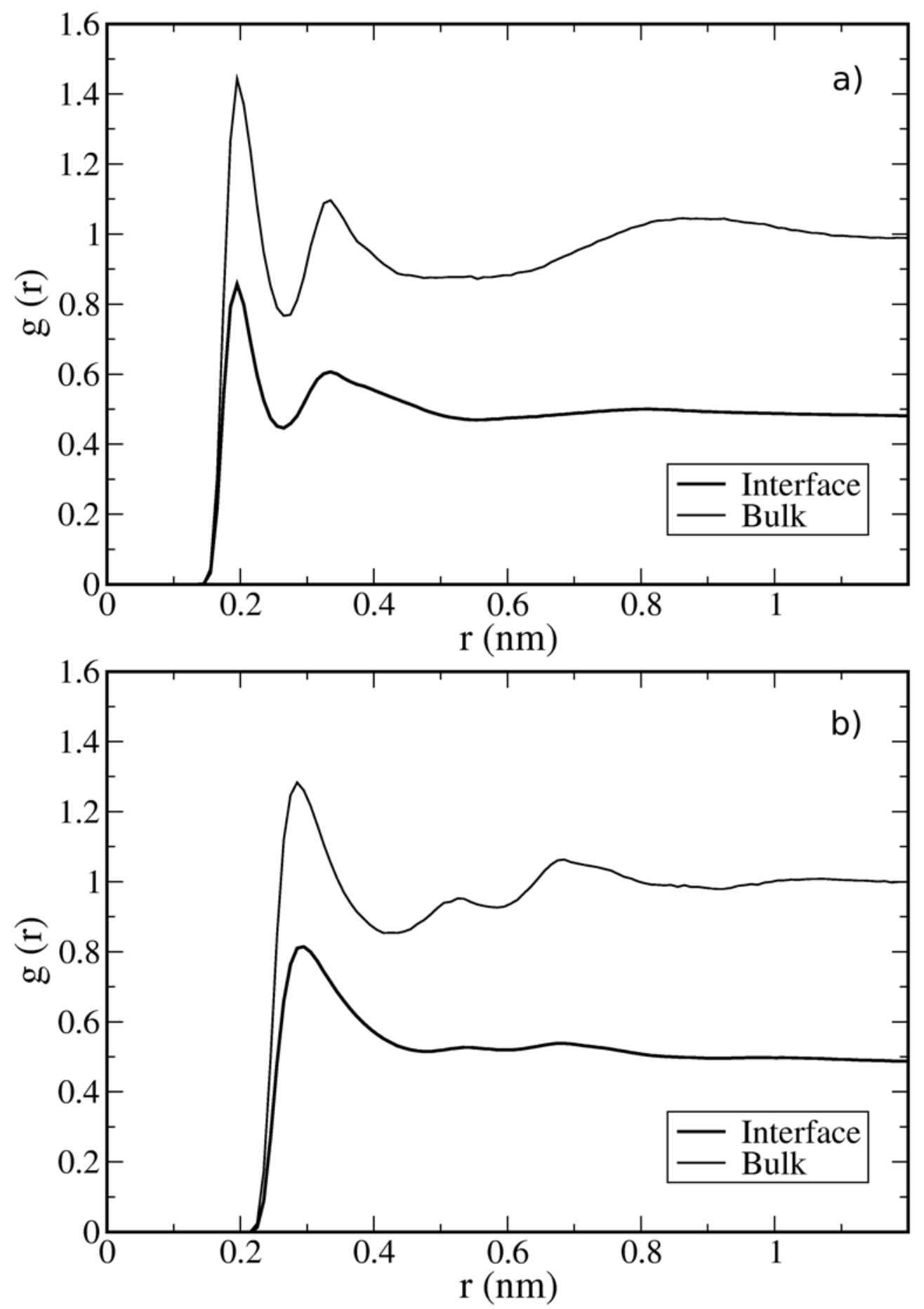

Figure 10. $\mathrm{H}_{\mathrm{W}}-\mathrm{O}_{\mathrm{N}}$ (a) and $\mathrm{O}_{\mathrm{W}}-\mathrm{H}_{\mathrm{A}}$ (b) $\mathrm{RDF}$ for the system water/MB at $L=3.5 \mathrm{~nm}$ in the interface and in bulk. 

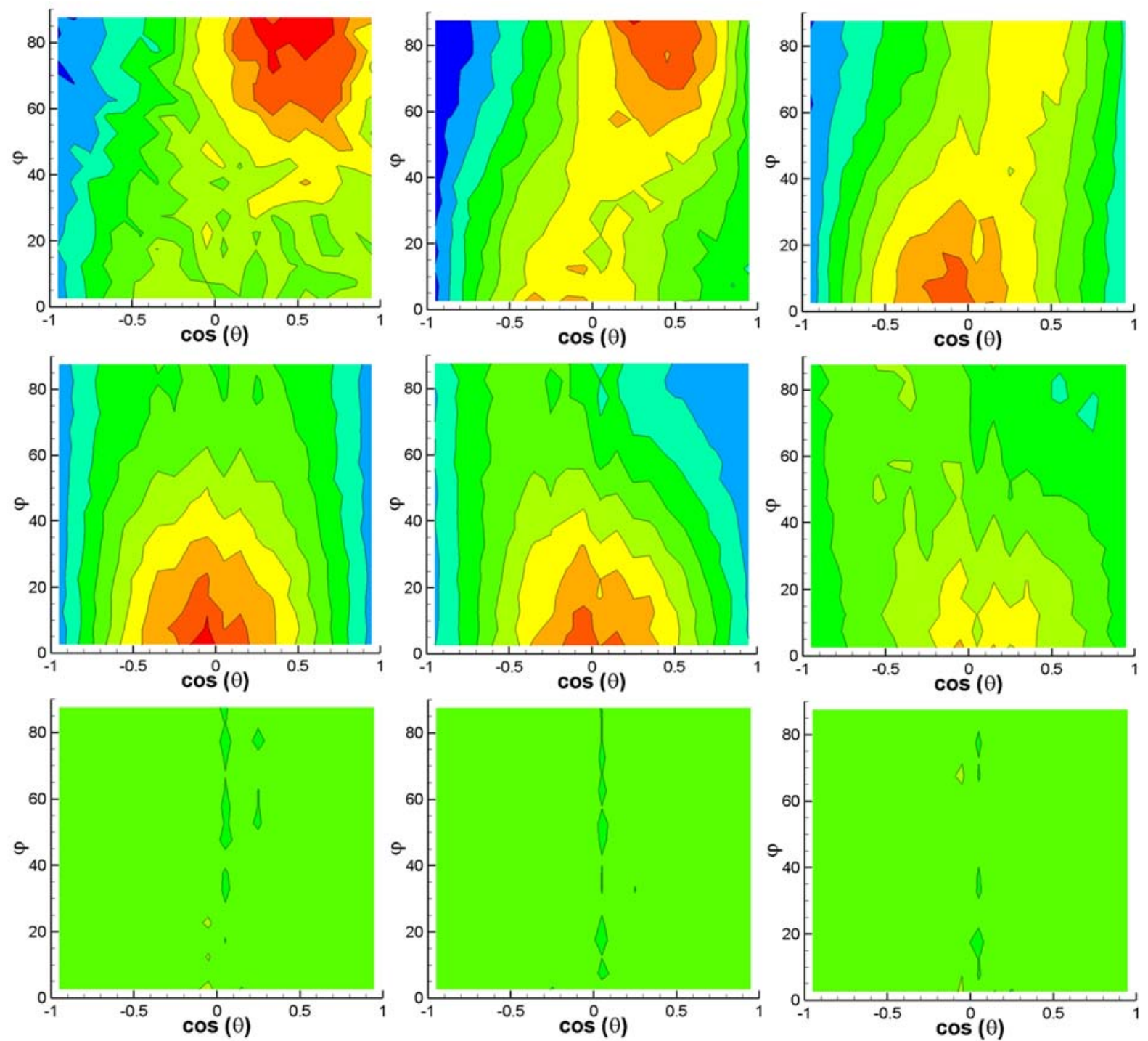

Figure 11. Bivariate orientation distribution of water molecules for the system water/MB at $L=3.5 \mathrm{~nm}$ calculated in slices perpendicular to the interface using a global definition $(N=1)$. Red corresponds to high normalized probability and blue to low probability. Slices, from top to bottom, correspond to positions relative to the limit of the organic phase of: $-0.9,-0.7,-0.5,-0.3,-$ $0.1,0.1,0.3,0.5$ and 0.7 . 

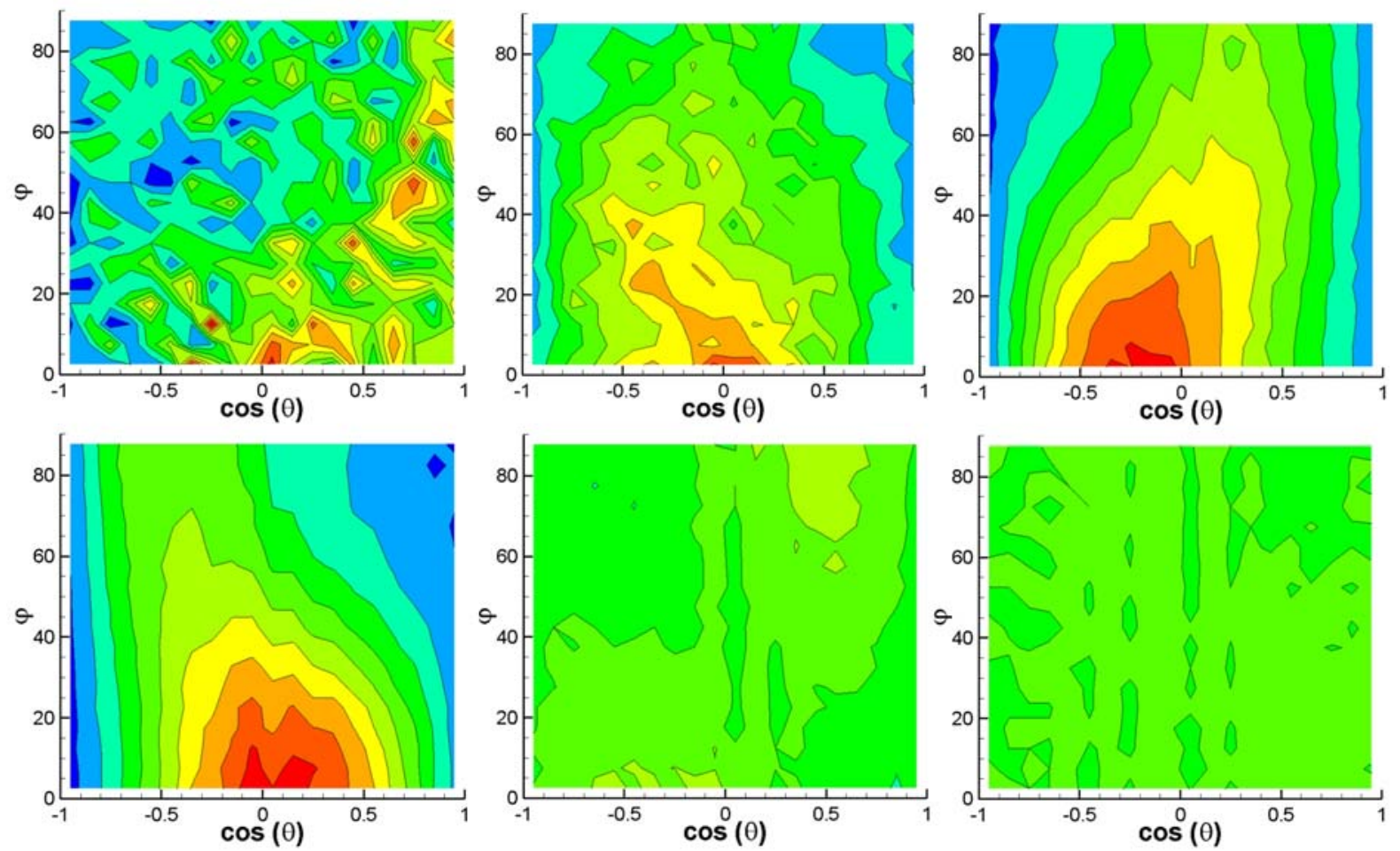

Figure 12. Bivariate orientation distribution of water molecules for the system water/MB at $L=3.5 \mathrm{~nm}$ calculated in slices perpendicular to the interface using a local definition $(N=10)$. Red corresponds to high normalized probability and blue to low probability. Slices, from top to bottom, correspond to positions relative to the limit of the organic phase of: $-0.3,-0.1,0.1,0.3$, 0.5 and 0.7 (slices closer to the organic phase are not statistically meaningful). 

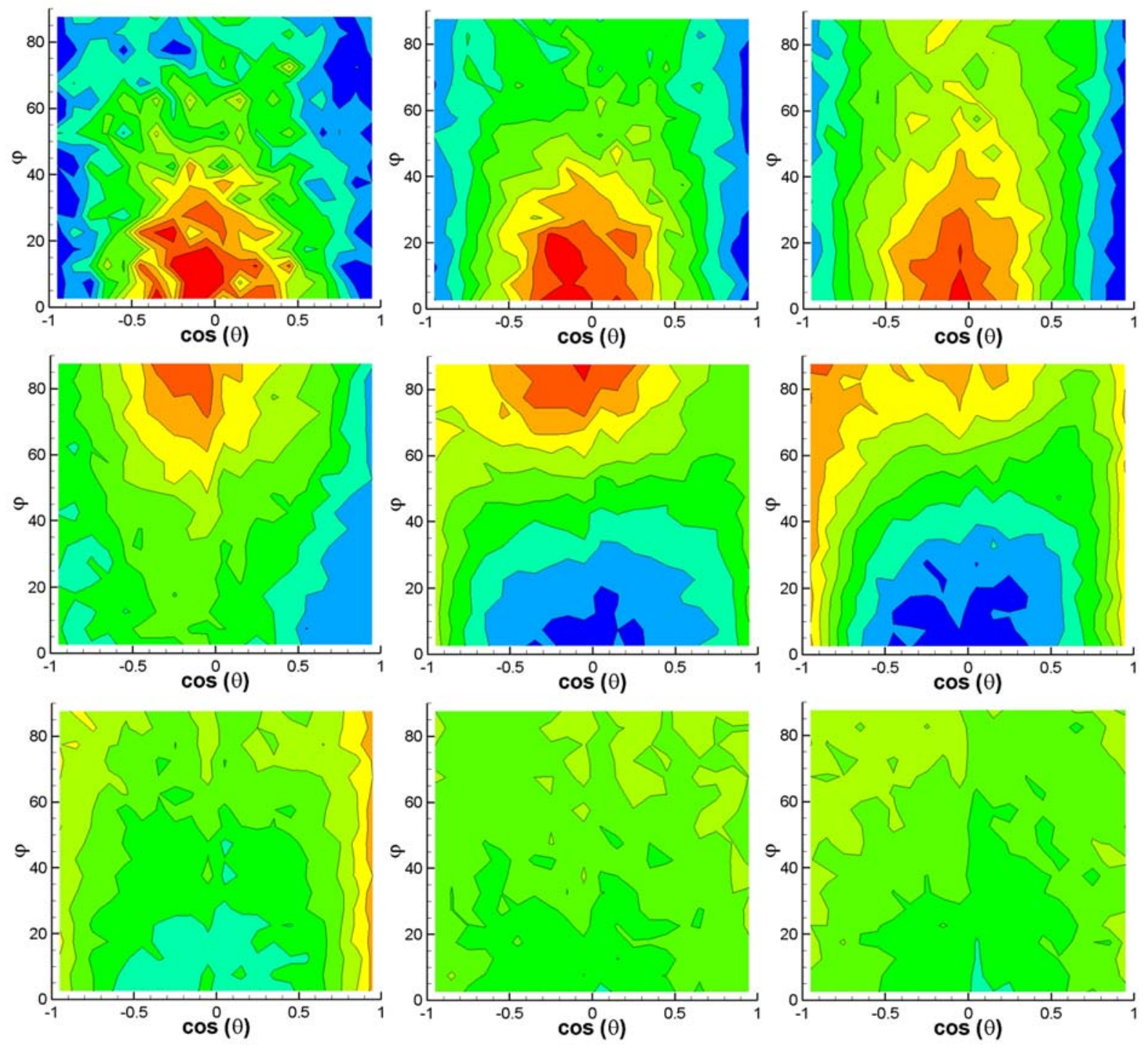

Figure 13. Bivariate orientation distribution of nitrobenzene molecules for the system water/MB at $L=3.5 \mathrm{~nm}$ calculated in slices perpendicular to the interface using a global definition $(N=1)$.

Red corresponds to high normalized probability and blue to low probability. Slices, from top to bottom, correspond to positions relative to the limit of the organic phase of: $-0.9,-0.7,-0.5,-0.3,-$ $0.1,0.1,0.3,0.5$ and 0.7 . 

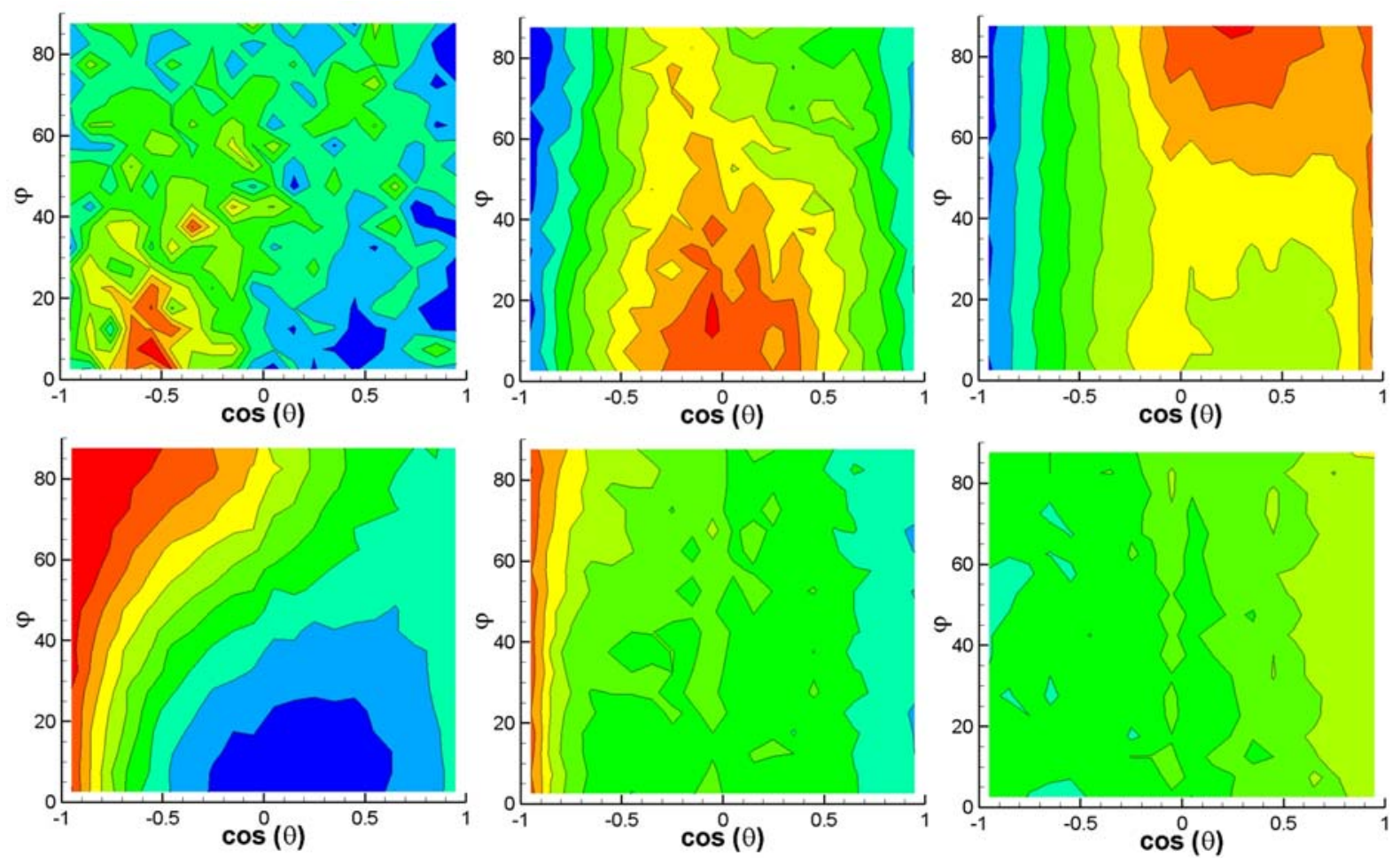

Figure 14. Bivariate orientation distribution of nitrobenzene molecules for the system water/MB at $L=3.5 \mathrm{~nm}$ calculated in slices perpendicular to the interface using a local definition $(N=11)$. Red corresponds to high normalized probability and blue to low probability. Slices, from top to bottom, correspond to positions relative to the limit of the organic phase of: $-0.1,0.1,0.3,0.5,0.7$ and 0.9 (slices closer to the water phase are not statistically meaningful). 


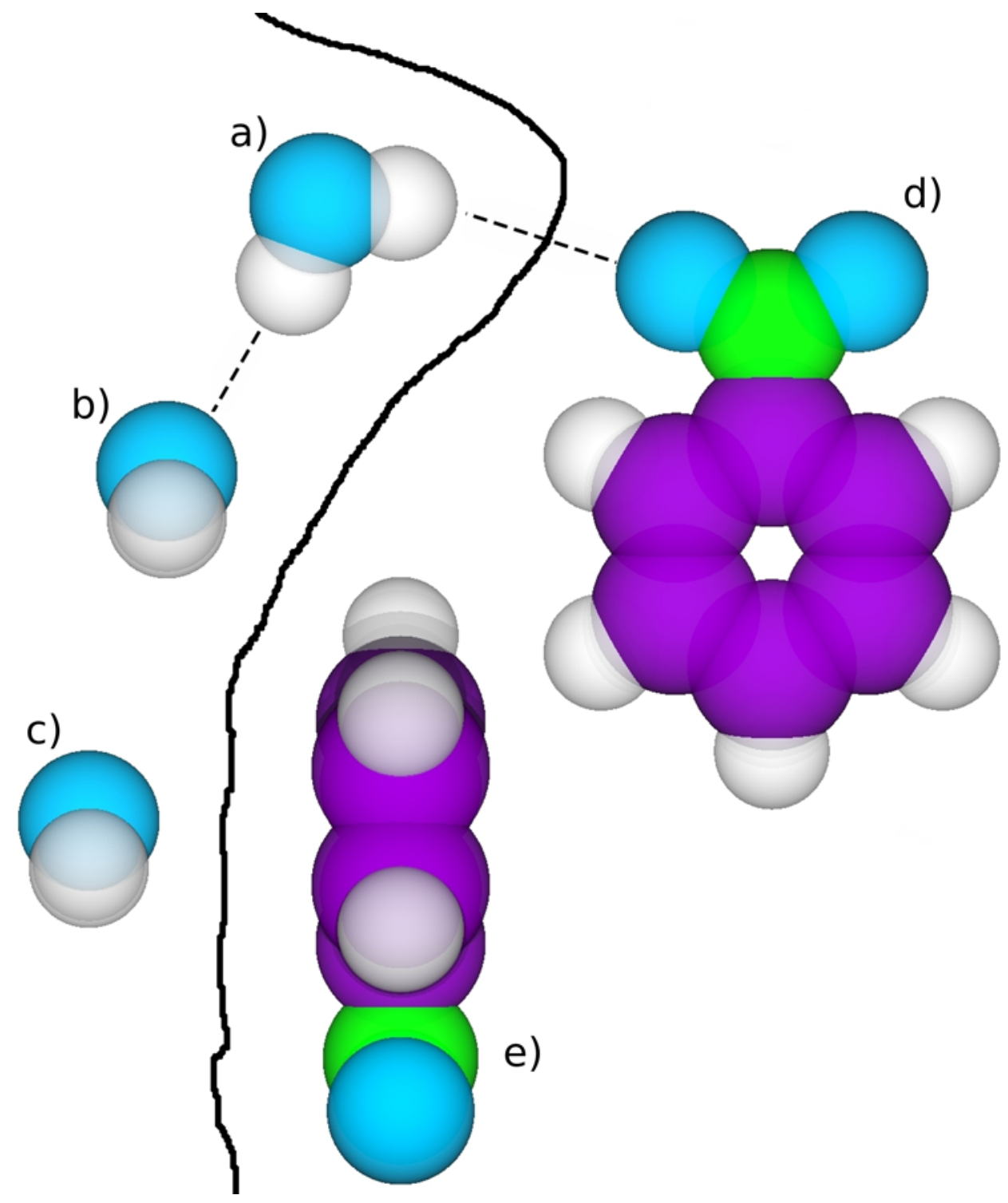

Figure 15. Schematic representation of the structure of the water/nitrobenzene interface. The diagram shows a hypothetical cross section through the $y z$ plane (i.e., perpendicular to the interfacial plane). Color coding is the same as in Figure 1. The thick line represents the interface and the thin dashed lines represent hydrogen bonds. 

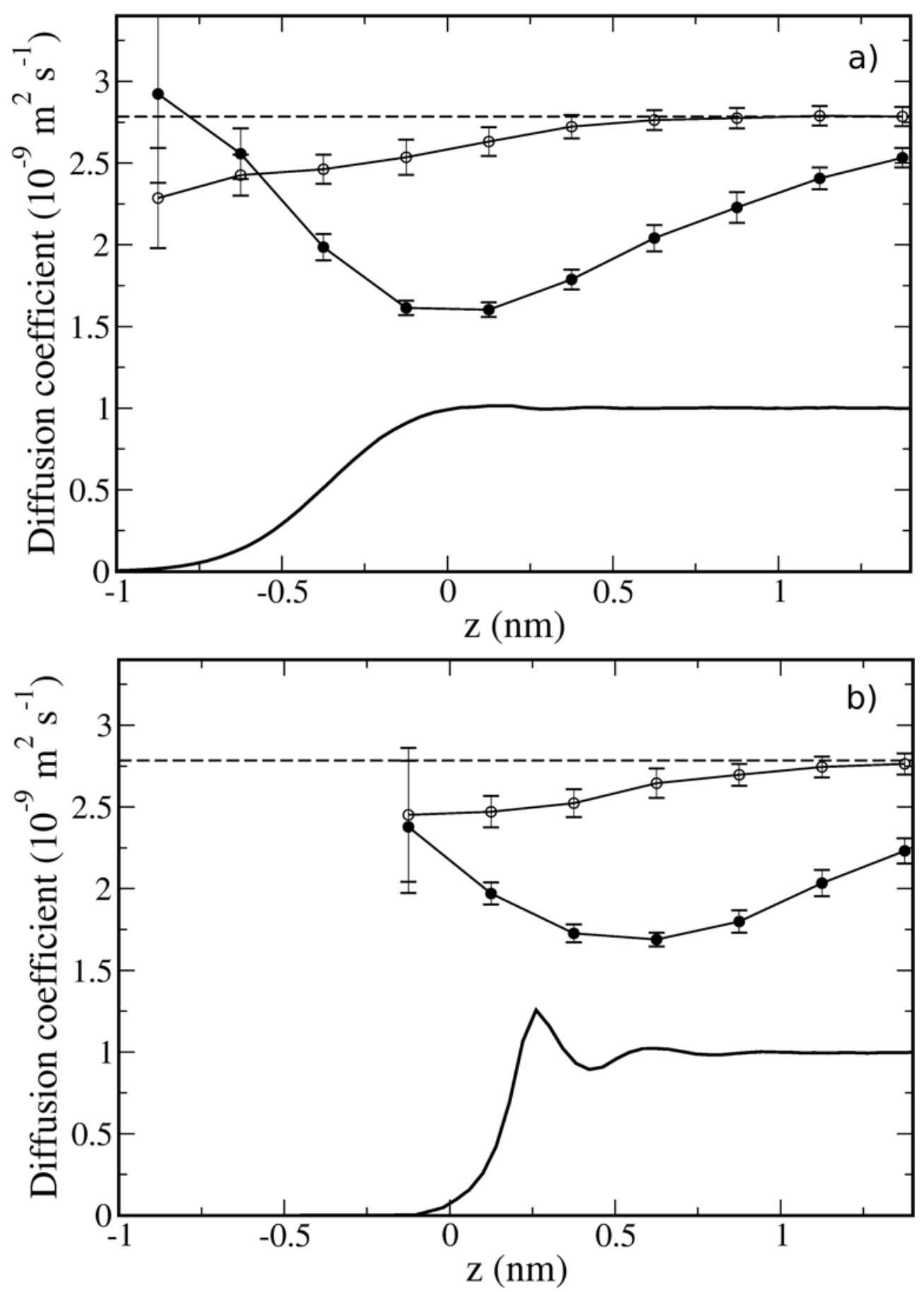

Figure 16. Water diffusion coefficient for the system water/MB at $L=3.5 \mathrm{~nm}$ calculated in slices perpendicular to the interface, defined globally (a) and locally (b). Open symbols are for diffusion in the plane of the interface, closed symbols are for diffusion perpendicular to the interface and the dashed line is the pure-component diffusion coefficient. The density profiles (thick lines) are superimposed for ease of visualization. 

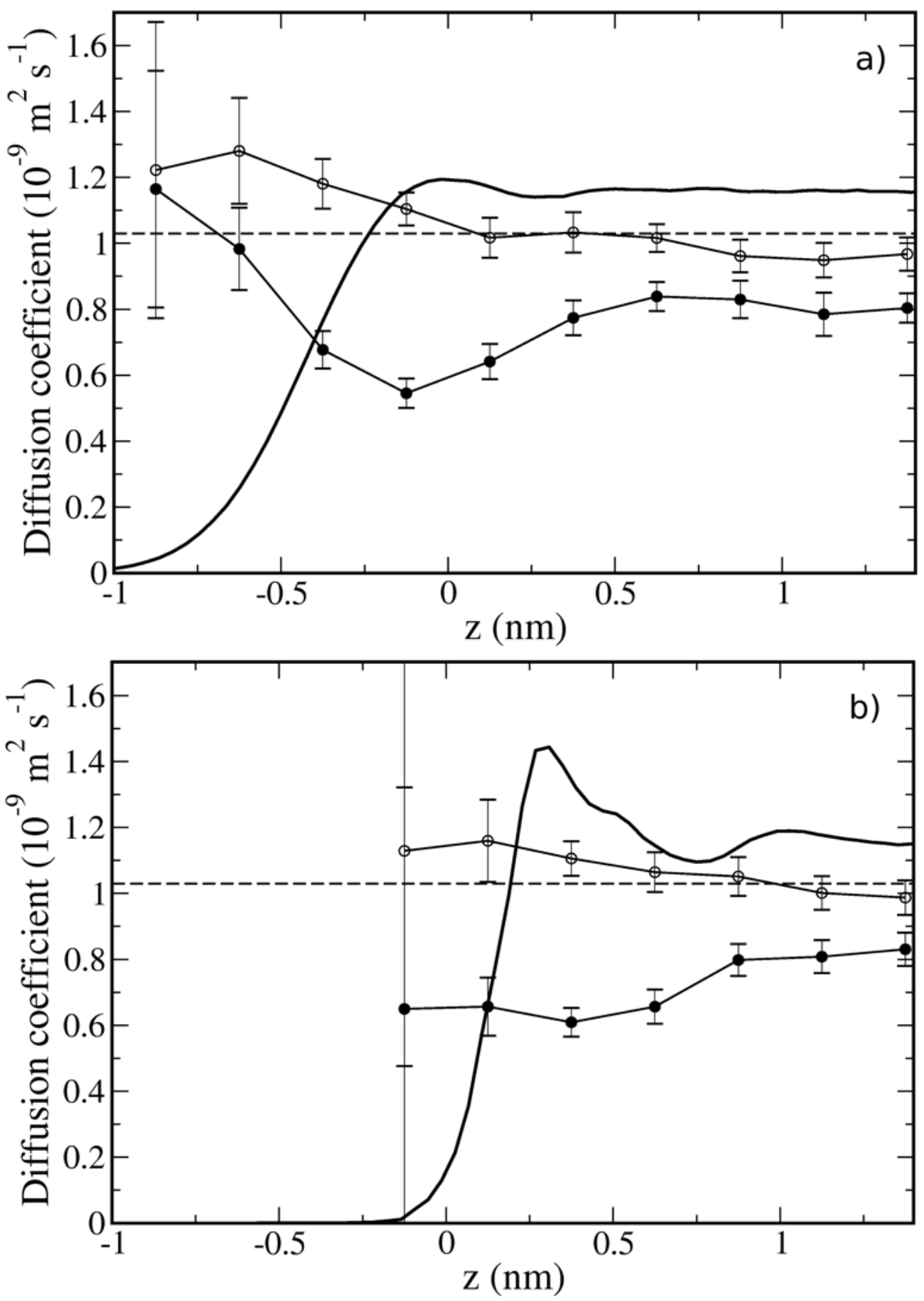

Figure 17. Nitrobenzene diffusion coefficient for the system water/MB at $L=3.5 \mathrm{~nm}$ calculated in slices perpendicular to the interface, defined globally (a) and locally (b). Open symbols are for diffusion in the plane of the interface, closed symbols are for diffusion perpendicular to the interface and the dashed line is the pure-component diffusion coefficient. The density profiles (thick lines) are superimposed for ease of visualization. 